\title{
Diatomáceas perifíticas em um sistema eutrófico brasileiro (Reservatório do Iraí, estado do Paraná)
}

\author{
Angela Maria da Silva ${ }^{1,3}$, Thelma Alvim Veiga Ludwig ${ }^{1}$, Priscila Izabel Tremarin ${ }^{1}$ e Ilka Schincariol Vercellino ${ }^{2}$
}

Recebido em 28/09/2009. Aceito em 7/10/2010

\begin{abstract}
RESUMO - (Diatomáceas perifíticas em um sistema eutrófico brasileiro (reservatório do Iraí, estado do Paraná)). O estudo sobre a diversidade das diatomáceas do reservatório do Iraí ampliou o conhecimento da flora diatomológica perifítica de ambientes eutróficos do estado do Paraná e contribuiu para subsidiar futuros trabalhos que utilizem diatomáceas como bioindicadoras das condições tróficas da água. Coletas trimestrais foram realizadas no reservatório durante um ano. As amostras perifíticas foram obtidas pela remoção do biofilme aderido a caules da macrófita Polygonum sp. e a lâminas de vidro. Estas foram colocadas em complexos de madeira e deixadas submersas durante 30 dias para colonização. O estudo taxonômico resultou na identificação de 11 citações pioneiras para o estado do Paraná entre as 96 espécies de diatomáceas determinadas. Maior diversidade de espécies foi registrada para o substrato macrófita. Achnanthidium minutissimum foi dominante na maior parte das amostras analisadas, sendo considerada tolerante às condições eutróficas do reservatório do Iraí. Fragilaria rumpens, Fragilaria vaucheriae var. capitellata e Gomphonema gracile foram abundantes. Informações ecológicas sobre as espécies foram obtidas em literatura, sendo 35\% características de sistemas aquáticos em processo avançado de eutrofização, $18 \%$ de ambientes mesotróficos e 19\% classificadas como tolerantes e indiferentes ao estado trófico dos ambientes.
\end{abstract}

Palavras-chave: algas, Bacillariophyta, bioindicador, eutrofização, perifíton

\begin{abstract}
Periphytic diatoms in a Brazilian eutrophic system (Iraí reservoir, Paraná state)). This floristic survey contributed to our knowledge of periphytic diatoms in a eutrophic reservoir in Paraná state, and provided a basis for future works using diatoms as bioindicators of trophic water conditions. Samples were collected quarterly, over a year, by removing the biofilm attached to Polygonum sp. stems and to glass slides. The slides were placed in wooden frames and remained submersed for 30 days to colonize. The taxonomic study resulted in 11 new taxonomic records for Paraná state among the 96 identified diatoms. The highest species diversity was found in the macrophyte samples. Achnanthidium minutissimum was dominant in most samples analyzed and considered tolerant of the eutrophic conditions in Iraí reservoir. Fragilaria rumpens, Fragilaria vaucheriae var. capitellata and Gomphonema gracile were abundant. Ecological information on the species was obtained from the literature; $35 \%$ of the species were characteristic of aquatic systems in an advanced state of eutrophication, $18 \%$ of mesotrophic environments and $19 \%$ were classified as tolerant and indifferent to the trophic state of the environment.

Key words: algae, bioindicator, Bacillariophyta, eutrophication, periphytic
\end{abstract}

\section{Introdução}

As diatomáceas vêm fazendo parte da rotina de programas de biomonitoramento em vários países de regiões temperadas, tais como os da América do Norte de Potapova \& Charles (2007) e Europa de Stenger-Kovács et al. (2007), Torrisi et al. (2006), Ács et al. (2005) e Kelly et al. (1998). São algas unicelulares, cosmopolitas, com elevada biodiversidade, mas de taxonomia complexa. Possuem curto ciclo de vida e por isso respondem rapidamente às alterações ambientais (Stoermer \& Smol 1999, Round et al. 1990). Abundantes nos ecossistemas aquáticos, este grupo coloniza rapidamente os substratos e é facilmente coletado e armazenado em lâminas permanentes. Por estas vantagens, estão consolidando-se como excelentes bioindicadoras ambientais.

$\mathrm{O}$ monitoramento de comunidades biológicas específicas pode funcionar como importante ferramenta para sinalizar alterações das condições limnológicas e hídrológicas dos reservatórios. Resultado dos diversos estudos desenvolvidos em ambientes lóticos, o software Omnidia (Lecoint et al. 1993) integrou a maioria dos índices propostos para avaliar a qualidade trófica de rios baseando-se na composição e abundância das espécies de diatomáceas, destacando-se os europeus de Schiefele \& Shreiner (1991), de Kelly \& Whitton (1995), canadense de Rott et al. (1998) e o argentino de Gómez \& Licursi (2001). São poucos os estudos com aplicação de índices em ambientes lênticos. Ács et al. (2005) utilizaram índices propostos para rios em sistema lacustre e relataram que o Índice Biológico de Diatomáceas (IBD) de Lenoir e Coste foi o que melhor refletiu as condições mesotróficas do lago Velence, na Hungria. Um índice de diatomáceas para avaliar o estado trófico especificamente de lagos foi proposto por StengerKovács et al. (2007), com o qual obtiveram bons resultados para ambientes húngaros.

Diferentes substratos (naturais ou artificiais) tem sido utilizados em pesquisas sobre avaliação de produção primária, processos de sucessão e relações das espécies com o meio ambiente (Schwarzbold 1990; Kelly et al. 1998; Kiss et al. 2003).

O conhecimento da diatomoflórula e das condições físicas e químicas dos ecossistemas aquáticos brasileiros é determinante para que índices de diatomáceas possam ser gerados e aplicados com segurança. Para tanto, o conhecimento da flora por ecorregiões brasileiras (Dinnerstein 1995) possibilitaria o estabelecimento de critérios que viabilizassem a geração de índices de diatomáceas para o país. O uso destes índices permitiria um panorama rápido sobre o estado trófico da água e medidas mitigatórias imediatas para evitar efeitos deletérios em um sistema eutrofizado.

\footnotetext{
1 Universidade Federal do Paraná, Departamento de Botânica, Setor de Ciências Biológicas, Curitiba, PR, Brasil

2 Centro Universitário São Camilo, São Paulo, SP, Brasil

3 Autor para correspondência: angela_ecologia@yahoo.com.br
} 
Poucos são os trabalhos florísticos publicados sobre diatomáceas perifíticas de reservatórios brasileiros. A maioria trata de estudos sobre toda a comunidade algácea perifítica trazendo apenas uma lista com os nomes das espécies encontradas, além de dados de biomassa e de nutrientes no ambiente, tais como: Felisberto \& Rodrigues (2005). Salienta-se a importância da inclusão de ilustrações para os organismos inventariados.

Para o estado do Paraná, podem-se destacar os estudos taxonômicos mais recentes sobre diatomáceas perifíticas que incluem registros fotográficos e descrições de espécies, como os de Leandrini et al. (2003), Landucci \& Ludwig (2005), Ludwig et al. (2005), Ferrari \& Ludwig (2007) e Tremarin et al. (2008, 2009), além dos trabalhos de cunho mais ecológico, que citam listas de espécies como Fonseca \& Rodrigues (2005), Cetto et al. (2004) e Rodrigues \& Bicudo (2001).

Através do estudo qualitativo e quantitativo das diatomáceas perifíticas em subatrato natural e artificial, em um reservatório eutrófico paranaense, pretendeu-se selecionar espécies com potencial bioindicador do estado trófico deste sistema.

\section{Material e métodos}

O reservatório do Iraí está inserido na bacia hidrográfica do rio Iguaçu, um dos maiores do Estado. Pertence ao município de Pinhais e constitui em um importante sistema para o abastecimento público de água da região metropolitana de Curitiba.

As coletas foram trimestrais, de outubro de 2007 a agosto de 2008 em duas estações de coletas. O substrato artificial (lâminas de vidro) foi posicionado próximo à tomada d'água do reservatório, nas coordenadas $25^{\circ} 25^{\prime} 30.08^{\prime \prime} \mathrm{S}$ e $49^{\circ} 6^{\prime} 46.23^{\prime \prime} \mathrm{O}$, e as macrófitas Polygonum sp. foram coletadas próximo à margem leste da represa, nas seguintes coordenadas $25^{\circ} 30^{\prime} 24.48^{\prime \prime} \mathrm{S} 49^{\circ}$ e 1'30.82”O. As amostras foram coletadas em tréplicas, por meio de sorteio. O substrato artificial permaneceu no local durante 30 dias, em laminários fixos em caixotes de madeira e submersos a 30 $\mathrm{cm}$ de profundidade. Para confecção das lâminas, os substratos foram raspados com o auxílio de lâminas cortantes envoltas com papel alumínio, e o material foi diluído em solução Transeau (6:3:1) (Bicudo \& Menezes 2006). A preparação das lâminas seguiu a técnica de Simonsen (1974) modificada por Moreira-Filho (1981) na qual se utilizaram permanganato de potássio $\left(\mathrm{KMnO}_{4}\right)$ e ácido clorídrico $(\mathrm{HCl})$ no processo de oxidação da matéria orgânica.

Os dados físicos e químicos foram cedidos pelo Instituto Ambiental do Paraná (IAP) (dados não publicados) e Companhia de Saneamento do Paraná (SANEPAR) (dados não publicados), a partir dos quais foi calculado o índice de Carlson (1977) modificado por Toledo et al. (1983) para a classificação trófica do reservatório.

Os registros fotográficos foram obtidos em fotomicroscópio Olympus BX40 com câmera de captura DP71 acoplada. Para os registros pioneiros para o estado do Paraná foram utilizadas apenas obras publicadas. A morfologia das valvas das diatomáceas foi baseada em Barber \& Haworth (1981) e a identificação dos táxons baseou-se em obras clássicas e recentes entre elas Hustedt (1927-1966), Patrick \& Reimer (1966), Krammer \& LangeBertalot (1991a,b, 1986, 1988), Metzeltin \& Lange-Bertalot (1998, 2005, 2007), Levkov et al. (2007).

Descrições foram providenciadas para as primeiras citações paranaenses e para os táxons determinados em nível genérico; os demais táxons foram listados (tabela 2), acompanhados de dados complementares. Comentários taxonômicos foram adicionados, quando relevantes.

A análise quantitativa foi realizada em lâminas permanentes contando-se até 600 valvas e considerando critério de abundância e dominância segundo Lobo \& Leighton (1986).

Análise de similaridade de Jaccard foi utilizada para verificar a similaridade florística entre o substrato artificial e natural.
As amostras foram registradas no herbário da Universidade Federal do Paraná (UPCB) sob os números UPCB 63363 a 63366 (lâmina de vidro) e UPCB 63367 a 63370 (macrófita Polygonum sp.).

\section{Resultados e discussão}

Baseando-se em dados estimados para o fósforo, clorofila -a e transparência da coluna d'água, o reservatório do Iraí foi classificado como eutrófico nos meses de novembro/2007 e fevereiro/2008, tendendo a mesotrofia nos meses de maio e agosto de 2008. Dados físicos e químicos estão relacionados na tabela 1 .

Foram determinadas 96 espécies de diatomáceas, sendo 11 em nível genérico, nas amostras perifíticas no reservatório eutrófico do Iraí. Cinquenta e nove foram detectadas em substrato artificial (lâmina de vidro), sendo 17 exclusivas deste substrato. Sobre o substrato natural registraram-se 68 espécies, sendo 26 exclusivas da macrófita. Onze espécies foram pioneiras para o estado do Paraná. A listagem das espécies com dados morfométricos, merísticos, ecológios e de ocorrência estão compilados na tabela 2. A similaridade de Jaccard entre os substratos artifical e natural foi de $32 \%$. Embora a composição taxonômica tenha sido distinta, as espécies dominantes e abundantes foram coincidentes para ambos os substratos.

\section{Família STEPHANODISCACEAE}

Discostella stelligeroides (Hustedt) Houk \& Klee, Diat. Res.

19(2): 223, 2004.

Fig. 1-3

Valvas circulares; área central plana, ornamentada por um poro central de onde irradiam interestrias bifurcadas, formando estrias curtas; anel de fultopórtulas marginal inconspícuo; estrias marginais radiais, alternadas em curtas e longas. Diâmetro valvar: 7,20 -11,30 $\mu \mathrm{m} ; 15-24$ estrias marginais em $10 \mu \mathrm{m}$.

D. stelligeroides diferencia-se pelo padrão de estriação de $D$. stelligera a qual apresenta estrias marginais regularmente encurtadas e poro central isolado e rodeado por interestrias simples.

Classificada como indiferente em relação ao valor de $\mathrm{pH}$ por Håkansson (1993).

Espécie pioneira para o estado do Paraná.

Ocorrência nas amostras: UPCB 63363, UPCB 63364, UPCB 63365, UPCB 63366, UPCB 63367, UPCB 63368, UPCB 63369 e UPCB 63370.

\section{Família FRAGILARIOPHYCEAE}

Fragilaria capucina var. austriaca (Grunow) LangeBertalot, Nova Hedwigia 33: 748, 1980.

Fig. 4-5

Valvas linear-lanceoladas; extremidades capitadas; esterno da rafe estreito, lanceolado; área central romboidal, alcançando as margens; estrias paralelas intercaladas. Compr.:

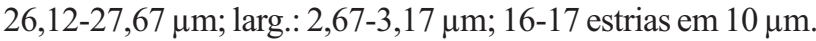


Tabela 1. Valores mínimos, máximos e desvio padrão dos dados físicos e químicos do Reservatório do Iraí estimados trimestralmente entre novembro de 07 e agosto de 2008 .

\begin{tabular}{|c|c|}
\hline Dados abióticos & Reservatório do Iraí \\
\hline Temperatura da água $\left({ }^{\circ} \mathrm{C}\right)$ & $\begin{array}{c}17,0-27,0 \\
(22,15 \pm 5,36)\end{array}$ \\
\hline Transparência (m) & $\begin{array}{c}0,7-1,1 \\
(0,95 \pm 0,17)\end{array}$ \\
\hline Condutividade elétrica $(\mu \mathrm{S} / \mathrm{cm})$ & $\begin{array}{c}48-67,3 \\
(56,07 \pm 8,34)\end{array}$ \\
\hline Turbidez (NTU) & $\begin{array}{c}6,0-17,3 \\
(9,6 \pm 5,30)\end{array}$ \\
\hline $\mathrm{pH}$ & $\begin{array}{c}7,65-8,17 \\
(7,83 \pm 0,24)\end{array}$ \\
\hline Nitrogênio Total (N - mg/L) & $\begin{array}{c}0,5-2,5 \\
(1,57 \pm 1,07)\end{array}$ \\
\hline Fósforo Total (P - mg/L) & $\begin{array}{c}0,03-0,04 \\
(0,03 \pm 0,005)\end{array}$ \\
\hline Alcalinidade Total (CaCO3 - mg/L) & $\begin{array}{l}19,6-24,64 \\
(21,7 \pm 2,36)\end{array}$ \\
\hline Demanda Química de Oxigênio - DQO ( mg/L) & $\begin{array}{c}20,0-48,0 \\
(37,1 \pm 13,04)\end{array}$ \\
\hline Demanda Bioquímica de Oxigênio $\mathrm{DBO}_{5}(\mathrm{mg} / \mathrm{L})$ & $\begin{array}{c}5,0-26,0 \\
(10,25 \pm 10,5)\end{array}$ \\
\hline Oxigênio Dissolvido (mg/L) & $\begin{array}{l}7,23-8,44 \\
(8,0 \pm 0,56)\end{array}$ \\
\hline
\end{tabular}

Fonte: IAP, SANEPAR (dados não publicados)

Tabela 2. Dados morfométricos, merísticos, ecológicos e de ocorrência nas amostras das diatomáceas perifíticas do reservatório do Iraí.

\begin{tabular}{|c|c|c|c|}
\hline Espécies & $\begin{array}{l}\text { Dimensões e } \\
\text { estrias (em } 10 \mu \mathrm{m}) \text { e outros }\end{array}$ & Dados ecológicos & Ocorrência nas amostras - UPCB \\
\hline \multicolumn{4}{|l|}{ THALASSIOSIRACEAE } \\
\hline $\begin{array}{l}\text { Thalassiosira rudis Tremarin, Ludwig, Beker \& Torgan } \\
\text { Fig. } 38-41\end{array}$ & $\begin{array}{l}\text { D: } 11,2-15,2 ; \mathrm{A}: 13-15 \text { em } 10 \mu \mathrm{m} ; \mathrm{Fu}: \\
4-5 \text { em } 10 \mu \mathrm{m} ; \mathrm{R}: 1-3\end{array}$ & & $\begin{array}{l}63363,63364,63365,63366 \\
63367,63368,63369,63370\end{array}$ \\
\hline \multicolumn{4}{|l|}{ STEPHANODISCACEAE } \\
\hline $\begin{array}{l}\text { Cyclotella meneghiniana Kützing } \\
\text { Fig. } 42-43\end{array}$ & D: $12,0-12,8 ; E: 9-10$ & eutrófico ${ }^{1}$ & $\begin{array}{l}63363,63364,63365,63367 \\
63368\end{array}$ \\
\hline $\begin{array}{l}\text { Discostella stelligera (Cleve \& Grunow) Houk \& Klee } \\
\text { Fig. } 44-46\end{array}$ & D: 12-21; E: 7,2-18,3 & $\begin{array}{l}\text { oligo-mesotróficos } \\
2,3\end{array}$ & $\begin{array}{l}63363,63364,63365,63366 \\
63367,63368,63369,63370\end{array}$ \\
\hline $\begin{array}{l}\text { Aulacoseira ambigua var. ambigua f. ambigua } \\
\text { (Grunow) Simonsen } \\
\text { Fig. } 47-49\end{array}$ & $\begin{array}{l}\text { D: 4,0-7,30; E: } 10-14 \text {; EP: } 15,17- \\
23,91 \mu \mathrm{m} ; \mathrm{A}: 10-14 \mathrm{em} 10 \mu \mathrm{m}\end{array}$ & $\begin{array}{l}\text { oligotrófico }{ }^{4}, \text { meso- } \\
\text { eutrófico }{ }^{1,5,6,7,8}\end{array}$ & $\begin{array}{l}63363,63364,63365,63366 \\
63367,63368,63369,63370\end{array}$ \\
\hline $\begin{array}{l}\text { Aulacoseira ambigua var. ambigua f. spiralis (Skuja) } \\
\text { Ludwig \& Valente-Moreira } \\
\text { Fig. } 50-51\end{array}$ & $\begin{array}{l}\text { D: 4,4-4,8; E: 12-16; EP: 19,64-21,68 } \\
\mu \mathrm{m} ; \mathrm{A}: 12-16 \text { em } 10 \mu \mathrm{m}\end{array}$ & & $\begin{array}{l}63363,63364,63365,63367 \\
63368,63369\end{array}$ \\
\hline $\begin{array}{l}\text { Aulacoseira granulata var. granulata (Ehrenberg) } \\
\text { Simonsen } \\
\text { Fig. } 52-54\end{array}$ & $\begin{array}{l}\text { D: 3,12-13,6; E: } 8-10 ; \text { EP: } 40-43,12 \\
\mu \mathrm{m} ; \mathrm{A}: 8-10 \text { em } 10 \mu \mathrm{m}\end{array}$ & eutrófico ${ }^{1}$ & $\begin{array}{l}63363,63364,63365,63366 \\
63367,63368,63369,63370\end{array}$ \\
\hline $\begin{array}{l}\text { Aulacoseira pusilla (Meister) Tuji \& Houki } \\
\text { Fig. } 55-59\end{array}$ & $\begin{array}{l}\text { D: } 5,47-6,4 \text {; estrias de difícil } \\
\text { contagem; EP: } 5,12-6,36 \mu \mathrm{m}\end{array}$ & eutrófico $^{9}$ & $\begin{array}{l}63363,63364,63365,63366 \\
63367,63368,63369,63370\end{array}$ \\
\hline $\begin{array}{l}\text { Aulacoseira tenella (Nygaard) Simonsen } \\
\text { Fig. } 60-61\end{array}$ & D: $6,08-6,85 ; A: 18-24$ em $10 \mu \mathrm{m}$ & & $\begin{array}{l}63364,63365,63367,63368 \\
63369\end{array}$ \\
\hline \multicolumn{4}{|l|}{ FRAGILARIOPHYCEAE } \\
\hline $\begin{array}{l}\text { Asterionella formosa Hassal } \\
\text { Fig. } 62-63\end{array}$ & $\begin{array}{l}\text { C: } 39,7-65,6 ; \mathrm{L}: 2,40-2,43 \text {; estrias } \\
\text { inconspícuas }\end{array}$ & meso-eutrófico ${ }^{1}$ & $\begin{array}{l}63363,63364,63365,63367 \\
63368,63369\end{array}$ \\
\hline
\end{tabular}


Tabela 2. Continuação.

\begin{tabular}{|c|c|c|c|}
\hline Espécies & $\begin{array}{l}\text { Dimensões e } \\
\text { estrias (em } 10 \mu \mathrm{m}) \text { e outros }\end{array}$ & Dados ecológicos & Ocorrência nas amostras - UPCB \\
\hline $\begin{array}{l}\text { Fragilaria crotonensis Kitton } \\
\text { Fig. } 64-66\end{array}$ & C: $48,8-72,0 ; \mathrm{L}: 2,4-2,8 ; \mathrm{E}: 12-17$ & mesotrófico $^{1}$ & $\begin{array}{l}63363,63364,63365,63366 \\
63367,63368,63369,63370\end{array}$ \\
\hline $\begin{array}{l}\text { Fragilaria gracilis Østrup } \\
\text { Fig. } 67-68\end{array}$ & C: $32,0-51,2 ; \mathrm{L}: 2,0-2,5 ; \mathrm{E}: 15-18$ & oligo-mesotrófico ${ }^{1}$ & $\begin{array}{l}63363,63364,63365,63366 \\
63367,63368,63369,63370\end{array}$ \\
\hline $\begin{array}{l}\text { Fragilaria javanica } \text { Hustedt } \\
\text { Fig. } 71-72\end{array}$ & C: $30,4-38,4 ;$ L: 5,6-6,4; E: 18-22 & & 63367 e 63368 \\
\hline $\begin{array}{l}\text { Fragilaria rumpens (Kützing) Carlson } \\
\text { Fig. } 75-77\end{array}$ & C: $20,0-39,8 ; \mathrm{L}: 2,4-2,9 ; \mathrm{E}: 12-17$ & tolerante $2,3,9,10$ & $\begin{array}{l}63363,63364,63365,63366 \\
63367,63368,63369,63370\end{array}$ \\
\hline $\begin{array}{l}\text { Fragilaria vaucheriae (Kützing) Petersen } \\
\text { Fig. } 69-70\end{array}$ & C: $13,0-24,0 ;$ L: $2,8-3,2 ; E: 12-15$ & meso-eutrófico ${ }^{1,10}$ & $\begin{array}{l}63363,63364,63365,63366 \\
63367,63368,63369,63370\end{array}$ \\
\hline $\begin{array}{l}\text { Fragilaria vaucheriae var. capitellata (Grunow) } \\
\text { Patrick } \\
\text { Fig. 73-74 }\end{array}$ & C: 9,6-25,6; L: 2,4-4,0; E: 12-14 & eutrófico $^{1}$ & $\begin{array}{l}63363,63364,63365,63367 \\
63368\end{array}$ \\
\hline $\begin{array}{l}\text { Synedra goulardii Brébisson ex Cleve \& Grunow } \\
\text { Fig. } 87\end{array}$ & C: 73,9; L: 9,1; E: 11 & & 63369 \\
\hline $\begin{array}{l}\text { Ulnaria delicatissima (Wm. Smith) Aboal \& Silva } \\
\text { Fig. } 89\end{array}$ & C: $62,4-80,4 ; \mathrm{L}: 2,40-3,2 ; \mathrm{E}: 14-16$ & $\begin{array}{l}\text { oligo-mesotrófico } \\
1,10\end{array}$ & 63363,63367 \\
\hline $\begin{array}{l}\text { Ulnaria ulna (Nitzsch) Compère } \\
\text { Fig. } 90-91\end{array}$ & C: 90,0-189,0; L: 4,0-4,8; E: 9-14 & $\begin{array}{l}\text { tolerante a } \\
\text { indiferente } \\
{ }^{1,10}\end{array}$ & $63363,63364,63365,63366$ \\
\hline \multicolumn{4}{|l|}{ EUNOTIACEAE } \\
\hline $\begin{array}{l}\text { Eunotia bilunaris (Ehrenberg) Mills } \\
\text { Fig. } 80\end{array}$ & C: $34,4-128,8 ; \mathrm{L}: 2,4-4,0 ; \mathrm{E}: 12-17$ & $\begin{array}{l}\text { tolerante a } \\
\text { indiferente } \\
{ }^{1,10}\end{array}$ & $63363,63365,63368$ \\
\hline $\begin{array}{l}\text { Eunotia camelus Ehrenberg } \\
\text { Fig. } 81-82\end{array}$ & $\begin{array}{l}\text { C: } 22,4-64,0 ; \mathrm{L}: 4,8-7,2 ; \mathrm{E}: 10-14 \\
\mathrm{O}: 2-4\end{array}$ & & $63364,63365,63367,63368$ \\
\hline $\begin{array}{l}\text { Eunotia didyma Grunow } \\
\text { Fig. } 92-93\end{array}$ & C: 61,$6 ; \mathrm{L}: 11,2 ; \mathrm{E}: 12 ; \mathrm{O}: 3$ & & 63365 \\
\hline $\begin{array}{l}\text { Eunotia flexuosa } \text { Brébisson ex. Kützing } \\
\text { Fig. } 95\end{array}$ & C: $88,0-149,6 ; \mathrm{L}: 4,8-5,6 ; \mathrm{E}: 14-19$ & oligotrófico ${ }^{1,10}$ & 63367,63368 \\
\hline $\begin{array}{l}\text { Eunotia major (Wm. Smith) Rabenhorst } \\
\text { Fig. } 96\end{array}$ & C: 89,$1 ; \mathrm{L}: 6,95 ; \mathrm{E}: 15$ & & 63365 \\
\hline $\begin{array}{l}\text { Eunotia minor (Kützing) Grunow } \\
\text { Fig. } 83-84\end{array}$ & C: 22,9-33,5; L: 4,7-5,8; E: 17-18 & tolerante ${ }^{1,10}$ & 63368 \\
\hline $\begin{array}{l}\text { Eunotia muscicola } \text { var. tridentula Nörpel } \\
\text { \& Lange-Bertalot } \\
\text { Fig. } 78\end{array}$ & $\begin{array}{l}\text { C: } 14,4-17,6 ; \mathrm{L}: 2,8-4,0 ; \mathrm{E}: 16-14 \\
\mathrm{O}: 3-4\end{array}$ & & 63365 \\
\hline $\begin{array}{l}\text { Eunotia naegelii Migula in Thomé } \\
\text { Fig. } 97\end{array}$ & C: $60,0-106,5 ; \mathrm{L}: 2,4-4,3 ; \mathrm{E}: 13-16$ & oligotrófico ${ }^{1,10}$ & 63364,63368 \\
\hline $\begin{array}{l}\text { Eunotia pirla } \text { Carter \& Flower } \\
\text { Fig. } 86\end{array}$ & C: 40,58; L: 7,0; E: 14 & & 63364 \\
\hline $\begin{array}{l}\text { Eunotia rabenhorstii Cleve \& Grunow } \\
\text { Fig. } 88\end{array}$ & C: $20,0-27,2 ; \mathrm{L}: 7,2-8,0 ; \mathrm{E}: 12-15$ & & $63363,63365,63367$ \\
\hline $\begin{array}{l}\text { Eunotia rhomboidea } \text { Hustedt } \\
\text { Fig. } 85\end{array}$ & C: $14,11-28,0 ; \mathrm{L}: 3,5-4,8 ; \mathrm{E}: 15-17$ & oligotrófico ${ }^{1,10}$ & 63367,63368 \\
\hline $\begin{array}{l}\text { Eunotia subarcuatoides Alles, Nörpel-Schempo } \\
\text { \& Lange-Bertalot } \\
\text { Fig. } 79\end{array}$ & C: 11,17; L: 3,47; E: 20 & oligotrófico $^{1}$ & 63368 \\
\hline $\begin{array}{l}\text { Eunotia sudetica O. Müller } \\
\text { Fig. } 98-99\end{array}$ & C: $16,8-17,6 ; \mathrm{L}: 4,0-4,8 ; \mathrm{E}: 15$ & oligo-mesotrófico ${ }^{1}$ & $63364,63365,63369$ \\
\hline CYMBELLACEAE & & & \\
\hline $\begin{array}{l}\text { Cymbella tumida (Brébisson) Van Heurck } \\
\text { Fig. } 105\end{array}$ & $\begin{array}{l}\text { C: } 64,0-80,0 ; \mathrm{L}: 16,0-21,6 ; \mathrm{E}: 7-12 \\
\text { dorsais; } 5-12 \text { ventrais }\end{array}$ & meso-eutrófico ${ }^{1,10}$ & $\begin{array}{l}63363,63364,63365,63367 \\
63368\end{array}$ \\
\hline $\begin{array}{l}\text { Cymbopleura naviculiformis (Auerswald) Krammer } \\
\text { Fig. } 94\end{array}$ & $\begin{array}{l}\text { C: } 32,0-33,6 ; \text { L: } 8,0-9,6 ; E: 12-14 \\
\text { dorsais; } 13-15 \text { ventrais }\end{array}$ & tolerante ${ }^{10}$ & $\begin{array}{l}63363,63364,63365,63367 \\
63368,63369,63370\end{array}$ \\
\hline $\begin{array}{l}\text { Encyonema minutum (Hilse) Mann } \\
\text { Fig. 107-108 }\end{array}$ & $\begin{array}{l}\text { C: } 19,2-21,6 ; \mathrm{L}: 6,4 ; \mathrm{E}: 9-13 \text { dorsais; } \\
\text { 12-15 ventrais }\end{array}$ & $\begin{array}{l}\text { oligo-mesotrófico } \\
1,10\end{array}$ & $\begin{array}{l}63363,63364,63365,63368 \\
63369,63370\end{array}$ \\
\hline
\end{tabular}


Tabela 2. Continuação.

\begin{tabular}{|c|c|c|c|}
\hline Espécies & $\begin{array}{l}\text { Dimensões e } \\
\text { estrias (em } 10 \mu \mathrm{m}) \text { e outros }\end{array}$ & Dados ecológicos & Ocorrência nas amostras - UPCB \\
\hline $\begin{array}{l}\text { Encyonema neomesianum Krammer } \\
\text { Fig. } 106\end{array}$ & $\begin{array}{l}\text { C: } 29,6-40,8 ; \text { L: } 8,0-11,2 ; \mathrm{E}: 9-10 \\
\text { dorsais; } 8-10 \text { ventrais }\end{array}$ & oligotrófico ${ }^{1,10}$ & 63367,63368 \\
\hline $\begin{array}{l}\text { Encyonema silesiacum (Bleisch) Mann } \\
\text { Fig. 109-110 }\end{array}$ & $\begin{array}{l}\text { C: } 42,4-54,4 ; \mathrm{L}: 11,2-12,0 ; \mathrm{E}: 8-11 \\
\text { dorsais; } 8-12 \text { ventrais }\end{array}$ & $\begin{array}{l}\text { tolerante a } \\
\text { indiferente } \\
\text { i,10 }\end{array}$ & $\begin{array}{l}63364,63365,63367,63368 \\
63369,63370\end{array}$ \\
\hline $\begin{array}{l}\text { Encyonopsis difficilis (Krasske) Krammer } \\
\text { Fig. } 111\end{array}$ & $\begin{array}{l}\mathrm{C}: 33,6 ; \mathrm{L}: 6,4 ; \mathrm{E}: 10 \text { dorsais; } 12 \\
\text { ventrais }\end{array}$ & oligotrófico ${ }^{11}$ & 63367 \\
\hline $\begin{array}{l}\text { Encyonopsis microcephala (Grunow) Krammer } \\
\text { Fig. 100-101 }\end{array}$ & C: 17,6-20,0; L: 3,9-4,3; E: 23-24 & meso-eutrófico ${ }^{1}$ & 63367,63370 \\
\hline $\begin{array}{l}\text { Encyonopsis schubartii (Hustedt) Krammer } \\
\text { Fig. } 102\end{array}$ & $\begin{array}{l}\text { C: } 26,4-27,2 ; \mathrm{L}: 6,4 ; \mathrm{E}: 10-12 \text { dorsais } \\
\text { e ventrais }\end{array}$ & oligotrófico ${ }^{11}$ & $63363,63367,63368$ \\
\hline \multicolumn{4}{|l|}{ GOMPHONEMATACEAE } \\
\hline $\begin{array}{l}\text { Gomphonema clevei Fricke } \\
\text { Fig. 13-14 }\end{array}$ & C: $32,8-37,6 ; \mathrm{L}: 4,8-7,2 ; \mathrm{E}: 11-15$ & & $\begin{array}{l}63363,63364,63365,63366 \\
63367\end{array}$ \\
\hline $\begin{array}{l}\text { Gomphonema gracile Ehrenberg } \\
\text { Fig. 103-104 }\end{array}$ & $\mathrm{C}: 21,0-54,4 ; \mathrm{L}: 4,0-8,8 ; \mathrm{E}: 11-17$ & mesotrófico ${ }^{1}$ & $\begin{array}{l}63363,63364,63365,63366 \\
63367,63368,63369,63370\end{array}$ \\
\hline $\begin{array}{l}\text { Gomphonema lagenula Kützing } \\
\text { Fig. } 114\end{array}$ & $\mathrm{C}: 17,6-24,8 ; \mathrm{L}: 4,8-7,2 ; \mathrm{E}: 11-16$ & tolerante ${ }^{11}$ & $\begin{array}{l}63363,63364,63365,63366 \\
63367,63368,63369,63370\end{array}$ \\
\hline $\begin{array}{l}\text { Gomphonema laticollum } \text { Reichardt } \\
\text { Fig. 116-117 }\end{array}$ & C: $34,4-46,4 ; \mathrm{L}: 10,4-12,8 ; \mathrm{E}: 10-13$ & & $\begin{array}{l}63363,63364,63365,63367 \\
63369,63370\end{array}$ \\
\hline $\begin{array}{l}\text { Gomphonema parvulum (Kützing) Kützing } \\
\text { Fig. 112-113 }\end{array}$ & $\mathrm{C}: 15,0-32,2 ; \mathrm{L}: 4,0-5,6 ; \mathrm{E}: 11-17$ & tolerante ${ }^{1,10}$ & $\begin{array}{l}63363,63364,63365,63366 \\
63367,63368,63369\end{array}$ \\
\hline $\begin{array}{l}\text { Gomphonema pseudoaugur Lange-Bertalot } \\
\text { Fig. } 118\end{array}$ & C: $24,8-28,0 ; \mathrm{L}: 4,8-5,6 ; \mathrm{E}: 12-16$ & eutrófico ${ }^{1,10}$ & 63363 \\
\hline $\begin{array}{l}\text { Gophonema pumilum (Grunow) Reichardt \& Lange- } \\
\text { Bertalot } \\
\text { Fig. } 115\end{array}$ & $\mathrm{C}: 13,4 ; \mathrm{L}: 3,9 ; \mathrm{E}: 13$ & eutrófico ${ }^{10}$ & 63363,63365 \\
\hline \multicolumn{4}{|l|}{ ACHNANTHACEAE } \\
\hline $\begin{array}{l}\text { Cocconeis placentula var. lineata (Ehrenberg) Van } \\
\text { Heurck } \\
\text { Fig. } 119\end{array}$ & C: 21,$65 ; \mathrm{L}: 11,7 ; \mathrm{E}: 23$ & eutrófico $^{1}$ & 63369 \\
\hline \multicolumn{4}{|l|}{ ACHNANTHIDIACEAE } \\
\hline $\begin{array}{l}\text { Achnanthidium minutissimum (Kützing) Czarnecki } \\
\text { Fig. 120-123 }\end{array}$ & $\begin{array}{l}\text { C: } 10,4-18,2 ; \mathrm{L}: 2,4-3,32 ; \text { estrias } \\
\text { inconspícuas }\end{array}$ & $\begin{array}{l}\text { tolerante a } \\
\text { indiferente } \\
\text { ind11 }\end{array}$ & $\begin{array}{l}63363,63364,63365,63366 \\
63367,63368,63369,63370\end{array}$ \\
\hline $\begin{array}{l}\text { Planothidium biporomum (Hohn \& Hellerman) } \\
\text { Lange-Bertalot } \\
\text { Fig. 124-125 }\end{array}$ & C: 12,$8 ; \mathrm{L}: 5,6 ; \mathrm{E}: 14$ & oligotrófico $^{1}$ & 63363 \\
\hline \multicolumn{4}{|l|}{ DIADESMIDIACEAE } \\
\hline $\begin{array}{l}\text { Diadesmis contenta (Grunow ex Van Heurck) Mann } \\
\text { Fig. 126-127 }\end{array}$ & $\begin{array}{l}\text { C: 9,6-10,4; L: 2,4-3,2; estrias } \\
\text { inconspícuas }\end{array}$ & indiferente ${ }^{1}$ & $\begin{array}{l}63364,63365,63367,63369 \\
63370\end{array}$ \\
\hline $\begin{array}{l}\text { Luticola mutica (Kützing) Mann } \\
\text { Fig. 128-129 }\end{array}$ & $\begin{array}{l}\text { C: } 16,0-27,2 ; \text { L: } 6,4-7,2 ; \mathrm{E}: 16-20 ; \mathrm{A}: \\
10 \mathrm{em} 10 \mu \mathrm{m}\end{array}$ & eutrófico ${ }^{1}$ & $63363,63364,63368,63369$ \\
\hline \multicolumn{4}{|l|}{ AMPHIPLEURACEAE } \\
\hline $\begin{array}{l}\text { Frustulia crassinervia (Brébisson) Costa } \\
\text { Fig. } 130\end{array}$ & $\begin{array}{l}\text { C: } 32,0-43,2 ; L: 8,0-9,6 \text {; estrias } \\
\text { delicadas }\end{array}$ & oligotrófico $^{1}$ & $\begin{array}{l}63363,63364,63365,63367 \\
63368,63369,63370\end{array}$ \\
\hline $\begin{array}{l}\text { Frustulia neomundana Lange-Bertalot \& Rumrich } \\
\text { Fig. 131-132 }\end{array}$ & C: 33,6-37,0; L: 8,0-8,8; E: 20 & & $\begin{array}{l}63363,63364,63365,63367 \\
63368,63369\end{array}$ \\
\hline $\begin{array}{l}\text { Frustulia saxonica } \text { Rabenhorst } \\
\text { Fig. } 133\end{array}$ & C: $36,8-42,4 ; \mathrm{L}: 8,8 ;$ estrias delicadas & oligotrófico $^{1}$ & 63367 e 63368 \\
\hline \multicolumn{4}{|l|}{ BRACHYSIRACEAE } \\
\hline $\begin{array}{l}\text { Brachysira neoexilis Lange-Bertalot } \\
\text { Fig. 134-135 }\end{array}$ & $\begin{array}{l}\text { C: } 23,2-26,4 ; \mathrm{L}: 4,8-5,6 ; \\
\text { estrias de difícil contagem }\end{array}$ & $\begin{array}{l}\text { oligotrófico a } \\
\text { mesotrófico }{ }^{12}\end{array}$ & $\begin{array}{l}63363,63364,63365,63366 \\
63367,63368,63369,63370\end{array}$ \\
\hline
\end{tabular}


Tabela 2. Continuação.

\begin{tabular}{|c|c|c|c|}
\hline Espécies & $\begin{array}{l}\text { Dimensões e } \\
\text { estrias }(\mathrm{em} 10 \mu \mathrm{m}) \text { e outros }\end{array}$ & Dados ecológicos & Ocorrência nas amostras - UPCB \\
\hline $\begin{array}{l}\text { Brachysira rostrata (Krasske) Metzeltin \& Lange- } \\
\text { Bertalot } \\
\text { Fig. } 136\end{array}$ & C: $53,6-60,0 ; \mathrm{L}: 12,8 ; \mathrm{E}: 19-22$ & oligotrófico $^{1}$ & 63363,63364 \\
\hline \multicolumn{4}{|l|}{ NEIDIACEAE } \\
\hline $\begin{array}{l}\text { Neidium affine (Ehrenberg) Pfitzer } \\
\text { Fig. } 137\end{array}$ & C: 37,6-40,0; L: 8,0-8,8; E: 22 & oligotrófico ${ }^{10}$ & 63367,63368 \\
\hline $\begin{array}{l}\text { Neidium ampliatum (Ehrenberg) Krammer } \\
\text { Fig. } 138\end{array}$ & $\mathrm{C}: 34,4-51,2 ; \mathrm{L}: 12,0-22,0 ; \mathrm{E}: 22-24$ & $\begin{array}{l}\text { oligo-mesotrófico } \\
1,10\end{array}$ & 63367,63368 \\
\hline \multicolumn{4}{|l|}{ PINNULARIACEAE } \\
\hline $\begin{array}{l}\text { Pinnularia borealis var. rectangularis Carlson } \\
\text { Fig. } 139\end{array}$ & $\mathrm{C}: 27,6-32,0 ; \mathrm{L}: 6,4-7,2 ; \mathrm{E}: 4-5$ & & 63366 \\
\hline $\begin{array}{l}\text { Pinnularia brauniana (Grunow) Mills } \\
\text { Fig. } 140\end{array}$ & $\mathrm{C}: 34,4-39,2 ; \mathrm{L}: 5,6-6,4 ; \mathrm{E}: 12$ & oligotrófico $^{1}$ & 63363 \\
\hline $\begin{array}{l}\text { Pinnularia divergens var. media Krammer } \\
\text { Fig. 141-142 }\end{array}$ & $\mathrm{C}: 34,0-48,0 ; \mathrm{L}: 8,0-10,0 ; \mathrm{E}: 14-16$ & oligotrófico $^{1}$ & $63367,63368,63369,3370$ \\
\hline $\begin{array}{l}\text { Pinnularia gibba } \text { Ehrenberg } \\
\text { Fig. 146-147 }\end{array}$ & $\mathrm{C}: 60,0-78,4 ; \mathrm{L}: 8,8-11,2 ; \mathrm{E}: 10-12$ & indiferente $^{1}$ & $\begin{array}{l}63363,63364,63365,63367 \\
63368,63369\end{array}$ \\
\hline $\begin{array}{l}\text { Caloneis bacillum (Grunow) Cleve } \\
\text { Fig. 148-149 }\end{array}$ & $\mathrm{C}: 22,4-35,2 ; \mathrm{L}: 6,4 ; \mathrm{E}: 20-22$ & meso-eutrófica & 63367,63368 \\
\hline $\begin{array}{l}\text { Chamaepinnularia mediocris (Krasske) Lange- } \\
\text { Bertalot } \\
\text { Fig. } 143\end{array}$ & $\mathrm{C}: 11,3 ; \mathrm{L}: 3,13 ; \mathrm{E}: 20$ & oligotrófico ${ }^{1,10}$ & 63368 \\
\hline \multicolumn{4}{|l|}{ DIPLONEIDACEAE } \\
\hline Diploneis ovalis (Hilse) Cleve Fig. 144 & $\begin{array}{l}\text { C: } 20,0-27,2 ; \mathrm{L}: 11,0-16,0 ; \mathrm{E}: 10-13 ; \\
\text { A: } 16 \mathrm{em} 10 \mu \mathrm{m}\end{array}$ & oligotrófico ${ }^{1,10}$ & $63367,63368,63369$ \\
\hline $\begin{array}{l}\text { Diploneis subovalis Cleve } \\
\text { Fig. } 145\end{array}$ & $\begin{array}{l}\mathrm{C}: 21,1-32,0 ; \mathrm{L}: 12,8-15,2 ; \mathrm{E}: 11 ; \mathrm{A}: \\
13-14 \mathrm{em} 10 \mu \mathrm{m}\end{array}$ & & 63366 \\
\hline \multicolumn{4}{|l|}{ NAVICULACEAE } \\
\hline $\begin{array}{l}\text { Navicula clementis var. linearis Brander ex Hustedt } \\
\text { Fig. } 150\end{array}$ & C: 30,0; L: 11,7; E: 23 & & 63367 \\
\hline $\begin{array}{l}\text { Navicula cryptocephala Kützing } \\
\text { Fig. } 151-152\end{array}$ & C: $23,2-39,2 ; \mathrm{L}: 4,8-8,8 ; \mathrm{E}: 12-16$ & $\begin{array}{l}\text { eutrófico a tolerante } \\
1,10\end{array}$ & $63363,63364,63365,63367$ \\
\hline $\begin{array}{l}\text { Navicula cryptotenella Lange-Bertalot } \\
\text { Fig. 154-155 }\end{array}$ & C: $20,0-30,4 ; \mathrm{L}: 4,8-5,6 ; \mathrm{E}: 12-14$ & $\begin{array}{l}\text { tolerante a } \\
\text { indiferente } \\
{ }^{1,10}\end{array}$ & $\begin{array}{l}63363,63364,63365,63366 \\
63367,63368,63369,63370\end{array}$ \\
\hline $\begin{array}{l}\text { Navicula leptostriata Jorgensen } \\
\text { Fig. } 157\end{array}$ & $\mathrm{C}: 33,0 ; \mathrm{L}: 5,0 ; \mathrm{E}: 17$ & oligo-mesotrófico ${ }^{1}$ & 63364 \\
\hline $\begin{array}{l}\text { Navicula rostellata Kützing } \\
\text { Fig. } 158\end{array}$ & C: 54,3; L: 8,2; E: 17 & eutrófico ${ }^{1,10}$ & 63367,63368 \\
\hline $\begin{array}{l}\text { Hippondonta avittata (Cholnoky) Lange-Bertalot } \\
\text { Fig. } 156\end{array}$ & $\mathrm{C}: 20,0 ; \mathrm{L}: 5,0 ; \mathrm{E}: 12$ & meso-eutrófico ${ }^{1}$ & 63367 \\
\hline \multicolumn{4}{|l|}{ STAURONEIDACEAE } \\
\hline Stauroneis gracilis Ehrenberg Fig. 160 & C: 47,7; L: 12,17; E: 22 & & 63368 \\
\hline $\begin{array}{l}\text { Stauroneis phoenicenteron (Nitzsch) Ehrenberg } \\
\text { Fig. } 161\end{array}$ & C: 87,$2 ; \mathrm{L}: 15,2 ; \mathrm{E}: 16$ & meso-eutrófico ${ }^{1}$ & 63368,63369 \\
\hline $\begin{array}{l}\text { Craticula halophila (Grunow ex Van Heurck) Mann } \\
\text { Fig. } 153\end{array}$ & $\mathrm{C}: 21,6 ; \mathrm{L}: 5,6 ; \mathrm{E}: 18$ & eutrófico ${ }^{1,10}$ & 63363 \\
\hline $\begin{array}{l}\text { Craticula riparia (Hustedt) Lange-Bertalot } \\
\text { Fig. } 159\end{array}$ & C: 37,55; L: 7,8; E: 21 & eutrófico ${ }^{1}$ & 63363 \\
\hline
\end{tabular}


Tabela 2. Continuação.

\begin{tabular}{|c|c|c|c|}
\hline Espécies & $\begin{array}{l}\text { Dimensões e } \\
\text { estrias (em } 10 \mu \mathrm{m}) \text { e outros }\end{array}$ & Dados ecológicos & Ocorrência nas amostras - UPCB \\
\hline \multicolumn{4}{|l|}{ BACILLARIACEAE } \\
\hline $\begin{array}{l}\text { Nitzschia clausii Hantzsch } \\
\text { Fig. } 164\end{array}$ & $\begin{array}{l}\text { C: } 39,2 ; \mathrm{L}: 3,2 ; \text { estrias inconspícuas; } \\
\text { F: } 12 \mathrm{em} 10 \mu \mathrm{m}\end{array}$ & eutrófico ${ }^{1,10}$ & 63363,63365 \\
\hline $\begin{array}{l}\text { Nitzschia gracilis Hantzsch } \\
\text { Fig. } 165\end{array}$ & $\begin{array}{l}\mathrm{C}: 36,0-60,0 ; \mathrm{L}: 2,4-4,0 ; \text { estrias } \\
\text { inconspícuas; F: } 10-15 \text { em } 10 \mu \mathrm{m}\end{array}$ & mesotrófico ${ }^{1}$ & $\begin{array}{l}63363,63364,63365,63367 \\
63368,63369\end{array}$ \\
\hline $\begin{array}{l}\text { Nitzschia palea (Kützing) Wm.Smith } \\
\text { Fig. } 166\end{array}$ & $\begin{array}{l}\text { C: } 14,6-39,6 ; \mathrm{L}: 2,0-4,0 ; \text { estrias } \\
\text { inconspícuas; F: } 9-13 \mathrm{~m} 10 \mu \mathrm{m}\end{array}$ & hipereutrófico ${ }^{1,10}$ & $\begin{array}{l}63363,63364,63365,63367 \\
63369\end{array}$ \\
\hline $\begin{array}{l}\text { Nitzschia perminuta (Grunow) Peragallo } \\
\text { Fig. } 167\end{array}$ & $\begin{array}{l}\text { C: } 25,6-27,2 ; \mathrm{L}: 2,4-3,2 ; \text { estrias } \\
\text { inconspícuas; F: 9-11 em } 10 \mu \mathrm{m}\end{array}$ & oligo-mesotrófico ${ }^{1}$ & 63367,63368 \\
\hline $\begin{array}{l}\text { Hantzschia amphioxys (Ehrenberg) Grunow } \\
\text { Fig. } 163\end{array}$ & $\begin{array}{l}\mathrm{C}: 49,6-65,7 ; \mathrm{L}: 6,4-6,5 ; \mathrm{E}: 22-23 ; \mathrm{F}: \\
10 \mathrm{em} 10 \mu \mathrm{m}\end{array}$ & indiferente ${ }^{1,10}$ & $63364,63368,63369$ \\
\hline \multicolumn{4}{|l|}{ RHOPALODIACEAE } \\
\hline $\begin{array}{l}\text { Rhopalodia gibberula var. vanheurckii O. Müller } \\
\text { Fig. } 162\end{array}$ & $\begin{array}{l}\text { C: } 22,0-40,8 ; \text { L: } 4,8-8,0 ; \text { E: } 15-20 \\
\text { CT: } 3-4 \text { em } 10 \mu \mathrm{m}\end{array}$ & & $\begin{array}{l}63363,63365,63367,63368 \\
63369,63370\end{array}$ \\
\hline \multicolumn{4}{|l|}{ SURIRELLACEAE } \\
\hline $\begin{array}{l}\text { Surirella angusta Kützing } \\
\text { Fig. } 168\end{array}$ & $\begin{array}{l}\mathrm{C}: 26,4-36,6 ; \mathrm{L}: 6,4-8,0 ; \mathrm{PA}: 6-8 \mathrm{em} \\
10 \mu \mathrm{m}\end{array}$ & eutrófica ${ }^{1,10}$ & $63367,63368,63370$ \\
\hline $\begin{array}{l}\text { Surirella linearis var. constricta Grunow } \\
\text { Fig. } 170\end{array}$ & $\begin{array}{l}\text { C: } 40,8-105,6 ; \mathrm{L}: 12,0-23,2 ; \mathrm{E}: 16-18 \\
\text { PA: } 2-4 \mathrm{em} 10 \mu \mathrm{m}\end{array}$ & oligo-mesotrófico ${ }^{1}$ & $63367,63368,63369$ \\
\hline $\begin{array}{l}\text { Surirella splendida (Ehrenberg) Kützing } \\
\text { Fig. } 171\end{array}$ & C: 113,$8 ; \mathrm{L}: 39,23 ; \mathrm{PA}: 2 \mathrm{em} 10 \mu \mathrm{m}$ & meso-eutrófico ${ }^{1}$ & 63367,63368 \\
\hline $\begin{array}{l}\text { Stenopterobia delicatissima (Lewis) Van Heurck } \\
\text { Fig. } 169\end{array}$ & $\begin{array}{l}\text { C: } 50,8 ; \mathrm{L}: 4,35 \\
\text { estrias inconspícuas; PA: } 6\end{array}$ & oligotrófico $^{1}$ & 63365 \\
\hline
\end{tabular}

${ }^{1}$ van Dam et al. (1994); ${ }^{2}$ Yang \& Dickman (1993); ${ }^{3}$ Potapova \& Charles (2007); ${ }^{4}$ Stenger-Kovács et al. (2007); ${ }^{5}$ Schiefele \& Schreiner (1991); ${ }^{6}$ Gómez \& Licursi (2001); ${ }^{7}$ Ivanov \& Kirilova (2004); ${ }^{8}$ Dela-Cruz et al. (2006); ${ }^{9}$ Tuji \& Williams (2007); ${ }^{10}$ Hofmann (1994); ${ }^{11}$ Krammer (2000); ${ }^{12}$ Lange-Bertalot (1994).

C: comprimento, L: largura, D: diâmetro e EP: eixo pervalvar, em $\mu \mathrm{m}$; A: aréolas, CT: costelas transapicais, F: fíbulas, Fu: fultopórulas, PA: processos aliformes e R: rimopórtulas, em $10 \mu \mathrm{m}$; O: ondulações, em toda a margem dorsal.

Espécie de água doce e salobra, de ambientes oligo-mesotróficos de $\mathrm{pH}$ alcalino (van Dam et al. 1994; Hofmann 1994).

Espécie pioneira para o estado do Paraná.

Ocorrência nas amostras: UPCB 63363, UPCB 63365, UPCB 63366, UPCB 63367, UPCB 63369 e UPCB 63370.

\section{Fragilaria sp.}

Fig. 6-7

Valvas lineares; extremidades capitadas, ápice arredondado; esterno da rafe estreito, linear; área central ampla, alcançando as margens; estrias alternadas. Compr.: 59,4874,19 $\mu \mathrm{m}$; larg.: 2,30-2,58 $\mu \mathrm{m}$; 16-17 estrias em $10 \mu \mathrm{m}$.

A população examinada assemelha-se a Synedra rumpens var. scotica Grunow e a Fragilaria familiaris (Kützing) Hustedt pelo formato delgado da valva e pela intumescência na região central. No entanto, são características ultraestruturais que melhor diferenciam as duas espécies, ou seja, a localização do poro de abertura da rimopórtula encontrase próximo a última estria em S.rumpens var. scotica e da penúltima em $F$. familiaris (Carneiro 2007).

Ocorrência nas amostras: UPCB 63364 e UPCB 63365.

\section{Família EUNOTIACEAE}

\section{Eunotia sp.}

Fig. 8

Valvas com margem dorsal convexa e ventral reta; extremidades subcapitadas, destacadas do corpo valvar, mais estreitas que a região mediana da valva; nódulos terminais próximos às extremidades; estrias radiadas em direção as extremidades, mais espaçadas na porção mediana da valva. Compr.: 54,41 $\mu \mathrm{m}$; larg.: $5 \mu \mathrm{m}$; 12 estrias em $10 \mu \mathrm{m}$.

Espécie semelhante à Eunotia veneris (Kützing) De Toni, porém esta última possui estrias eqüidistantes em todo corpo valvar, margem dorsal extremamente côncava com comprimento valvar menor e largura maior do que as estimadas para Eunotia sp. (Carter \& Flower, 1988).

Ocorrência nas amostras: UPCB 63365.

\section{Família CYMBELLACEAE}

Placoneis pseudoanglica (Lange-Bertalot) Cox, Diat. Res., 2(2): 155, fig. 11, 12, 17-19, 47, 49, 50, 1987. Fig. 9 
Valvas lanceoladas; extremidades rostradas; esterno da rafe linear, estreito; rafe linear, reta; área central atenuada, limitada pelo encurtamento irregular das estrias medianas; estrias radiadas ao longo da superfície valvar. Compr.: 20,0 $\mu \mathrm{m}$; larg.: 7,65 $\mu \mathrm{m} ; 16$ estrias em $10 \mu \mathrm{m}$.

Espécie de água doce e salobra, de pH alcalino, está presente em águas meso-eutróficas (Håkansson 1993; van Dam et al. 1994; Hofmann 1994).

Espécie pioneira para o estado do Paraná.

Ocorrência nas amostras: UPCB 63367.

Cymbella australica (Schimdt) Cleve, Sve. Vet. Akad. Handl. 26(2): 1-194, pl. 5, 1894.

Fig. 10

Valvas dorsiventrais; margem dorsal distintamente convexa; margem ventral delicadamente côncava nas extremidades e levemente convexa na região mediana; extremidades truncadas, podendo se apresentar arredondadas; esterno da rafe linear estreito; área central elíptica com um estigma ventral; rafe arqueada, extremidades distais fletidas dorsalmente e proximais voltadas ventralmente; estrias convergentes de aspecto grosseiro; aréolas conspícuas. Compr.: 120,0-139,6 $\mu \mathrm{m}$; larg.: 23,33-23,80 $\mu \mathrm{m}$; estrias medianas: $8-9 \mathrm{em} 10 \mu \mathrm{m}$; estrias nas extremidades: $10-12 \mathrm{em} 10 \mu \mathrm{m}$; 12-14 aréolas em $10 \mu \mathrm{m}$.

Diferencia-se de Cymbella charrua Metzeltin, LangeBertalot \& García-Rodríguez que apresenta medidas inferiores de comprimento e largura. Metzeltin, Lange-Bertalot \& García-Rodríguez (2005) ressaltaram que as aréolas de C. australica tem forâmen em forma de ferradura enquanto que $C$. charrua apresenta ramificações sob microscopia eletrônica de varredura.

Dados ecológicos não foram encontrados na literatura.

Espécie pioneira para o estado do Paraná.

Ocorrência nas amostras: UPCB 63363.

\section{Encyonema sp.}

Fig. 11-12

Valvas dorsiventrais; margem dorsal convexa; margem ventral convexa; extremidades atenuado-arredondadas, levemente voltadas para baixo; esterno da rafe linear, estreito; área central expandidda; estigmóide mediano dorsal; rafe curvada com a extremidade proximal fletida dorsalmente e distal ventralmente; estrias dorsais paralelas a radiadas; estrias ventrais paralelas a convergentes nas extremidades. Compr.: 25,6-31,2 $\mu \mathrm{m}$; larg.: 6,4-7,2 $\mu \mathrm{m}$; 13-15 estrias dorsais em $10 \mu \mathrm{m} ; 14-15$ estrias ventrais em $10 \mu \mathrm{m}$.

Semelhante a Encyonema riotecense Krammer do qual se diferencia pelos exemplares com estrutura mais delicada e dados morfométricos inferiores. Os indivíduos constatados por Krammer (1997a) e por Metzeltin \& Lange-Bertalot (1998) são maiores e mais grosseiros, apresentando respectivamente, compr. de 32-76 $\mu \mathrm{m}$ e larg. de 7,6-11 $\mu \mathrm{m}$ e compr. de 38,6 a $86,67 \mu \mathrm{m}$ e larg. de 9,3 a $14,67 \mu \mathrm{m}$ de largura e a densidade de estrias em ambas as obras é menor que Encyonema sp., 10-13 estrias em $10 \mu \mathrm{m}$.

Ocorrência nas amostras: UPCB 63364, UPCB 63365, UPCB 63367 e UPCB 63368.

\section{Família GOMPHONEMATACEAE}

\section{Gomphonema clevei Fricke obra}

Fig. 13-14

Destaca-se a presença de área axial com granulações na população de G. clevei do reservatório do Irai. Acredita-se que tal estrutra não justifique outra identidade taxonômica, pois a presença ou ausência de granulação ocorre em algumas outras espécies do gênero, como em G. gibberum Hustedt, G. butantanum Krasske (Metzeltin \& Lange-Bertalot, 1998; Simonsen, 1987). Formas com extremidades curvadas lateralmente foram registradas, tratando-se de deformidades estruturais.

Ocorrência nas amostras: UPCB 63363, UPCB 63364, UPCB 63365, UPCB 63366 e UPCB 63367.

\section{Gomphonema sp. 2}

Fig. 15-16

Valvas heteropolares lanceoladas; ápice e base afilados; esterno da rafe linear, estreito; rafe filiforme, reta e simples; estrias espaçadas, radiadas na porção central da valva e paralelas em direção as extremidades; região central é limitada por estrias medianas; estigma presente próximo ao nódulo central junto com a estria mediana encurtada. Compr.: 22,237,1 $\mu \mathrm{m}$; larg.: 4,4-7,3 $\mu \mathrm{m}$; 10-12 estrias em $10 \mu \mathrm{m}$.

O táxon assemelha-se a Gomphonema utae LangeBertalot \& Reichardt e Gomphonema bohemicum Reichelt \& Fricke nos dados morfométricos, porém G. utae apresenta valvas linear-elípticas e rafe discretamente sinuosa e $G$. bohemicum não apresenta estria mediana no lado oposto ao estigma (Reichardt, 1999, pl. 28, fig. 1-37; pl. 29, fig. 1-7; pl. 61, fig. 1-22; pl. 63, fig 1-5).

Ocorrência nas amostras: UPCB 63363, UPCB 63364, UPCB 63365, UPCB 63366 e UPCB 63367.

\section{Família SELLAPHORACEAE}

Sellaphora densistriata (Lange-Bertalot \& Metzeltin) Lange-Bertalot \& Metzeltin, Iconogr. Diatomol. 11: 58, 2002.

Fig. 17-18

Valvas lanceoladas; extremidades capitadas, amplamente arredondadas; esterno da rafe linear, estreito; área central expandida, limitada pelo encurtamento irregular das estrias medianas; rafe filiforme, extremidades proximais discretamente fletidas para um dos lados; estrias radiadas ao longo da superfície valvar, mais espaçadas na porção mediana. Compr.: 24,34-26,5 $\mu \mathrm{m}$; larg.: 7,30-7,38 $\mu \mathrm{m}, 24-25$ estrias em $10 \mu \mathrm{m}$.

Espécie de água doce e salobra, meso-eutrófica de $\mathrm{pH}$ neutro (van Dam et al. 1994). 

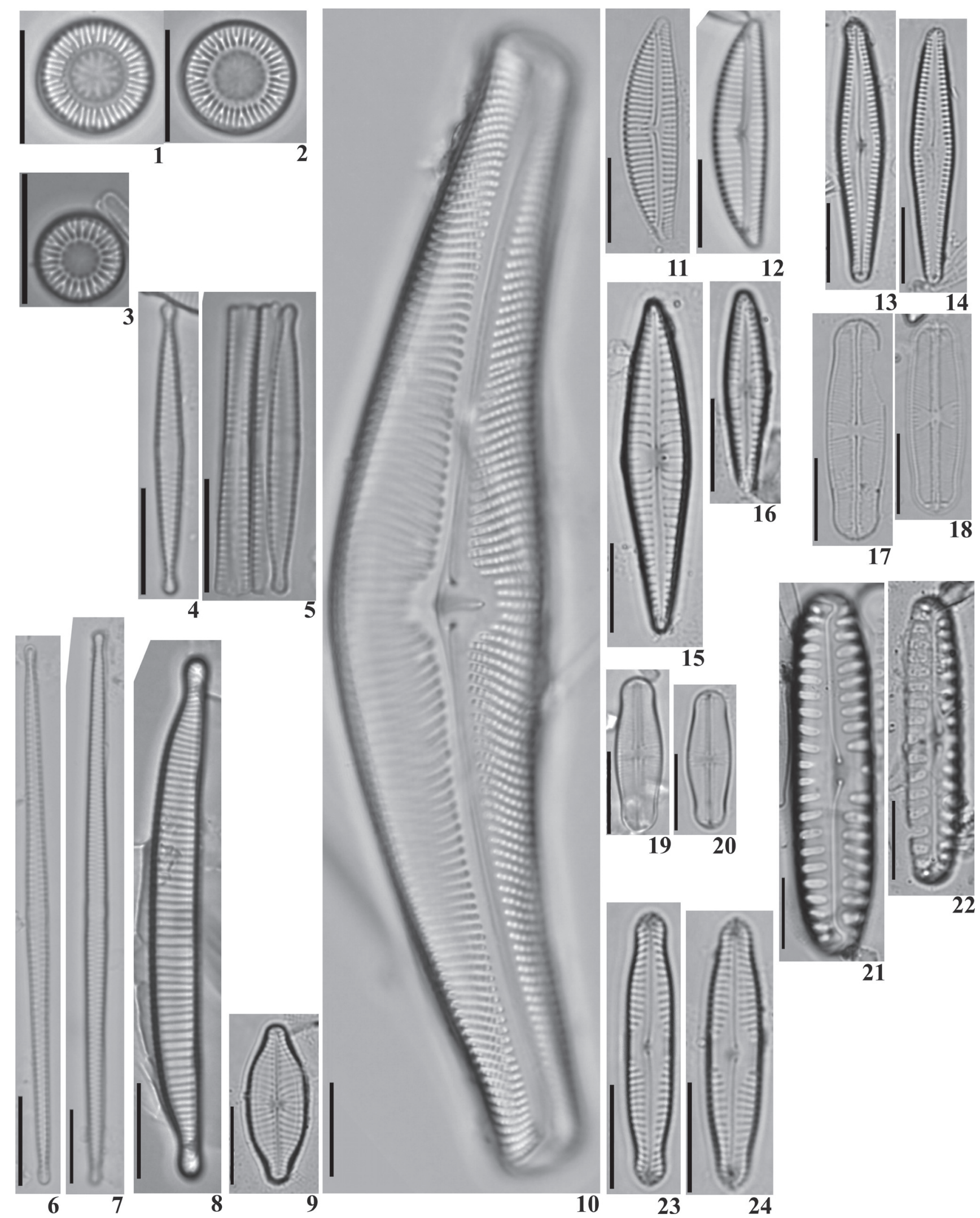

Figuras 1-24. Diatomáceas perifíticas do reservatório eutrófico do Iraí. 1-3 Discostella stelligeroides (Hustedt) Houk \& Klee; 4-5 Fragilaria capucina var. austriaca (Grunow) Lange-Bertalot; 6-7 Fragilaria sp.; 8 Eunotia sp.; 9 Placoneis pseudoanglica (Lange-Bertalot) Cox; 10 Cymbella australica (Schimdt) Cleve; 11-12 Encyonema sp.; 13-14 Gomphonema sp. 1; 15-16 Gomphonema sp. 2; 17-18 Sellaphora densistriata (Lange-Bertalot \& Metzeltin) Lange-Bertalot \& Metzeltin; 19-20 Sellaphora sp.; 21-22 Pinnularia borealis var. scalaris (Ehrenberg) Rabenhorst; 23-24 Pinnularia microstauron var. rostrata Krammer. Escala: 10 um. 
Espécie pioneira para o estado do Paraná.

Ocorrência nas amostras: UPCB 63365 e UPCB 63367.

\section{Sellaphora sp.}

Fig. 19-20

Valvas lanceoladas; extremidades capitadas, amplamente arredondadas; esterno da rafe linear, estreito; área central expandida, limitada pelo encurtamento de duas estrias medianas; rafe filiforme, simples; estrias delicadas, radiadas ao longo da superfície valvar, mais espaçadas na porção mediana. Compr.: 18,4-28,0 $\mu \mathrm{m}$; larg.: 5,6-7,2 $\mu \mathrm{m}, 16-22$ estrias em $10 \mu \mathrm{m}$.

Não foi encontrado na literatura espécie de Sellaphora que apresentasse formato valvar semelhante e características valvares tão delicadas quanto às encontradas nos exemplares analisados.

Ocorrência nas amostras: UPCB 63363, UPCB 63364, UPCB 63365, UPCB 63367 e UPCB 63368.

\section{Família PINNULARIACEAE}

Pinnularia borealis var. scalaris (Ehrenberg) Rabenhorst, Flora Eur. Alg., p. 216, 1864.

Fig. 21-22

Valvas lineares eliptícas; extremidades arredondadas; margens paralelas; esterno da rafe linear, estreito; área central circular, limitada pelo encurtamento de uma ou duas estrias centrais; rafe filiforme, extremidades proximais fletidas na mesma direção; extremidades distais em forma de foice, rvadas em direção opostas a extremidade proximal; estrias grosseiras, radiais na porção mediana da valva e paralelas em direção as extremidades. Compr.: 24,77 $\mu \mathrm{m}$; larg.: 6,0 $\mu \mathrm{m} ; 5$ estrias em $10 \mu \mathrm{m}$.

Não foram encontrados dados ecológicos na literatura.

Espécie pioneira para o estado do Paraná.

Ocorrência nas amostras: UPCB 63366.

Pinnularia microstauron var. rostrata Krammer, Diat. Eur., 1: 74, 217, pl. 51, fig. 8-18, 2000.

Fig. 23-24

Valvas lineares; extremidades rostradas; esterno da rafe linear-lanceolado, alargando-se em direção a área central; margens paralelas com duas discretas ondulações; área central romboidal formando uma fáscia bilateral; rafe filiforme com as extremidades proximais fletidas para o mesmo lado; extremidades distais em forma de foice; estrias radiadas na porção mediana e convergentes em direção as extremidades. Compr.: 24,0-30,4 $\mu \mathrm{m}$; larg.: 5,0-5,6 $\mu \mathrm{m}$; 14-16 estrias em $10 \mu \mathrm{m}$.

Espécie de água doce, oligotrófica, águas ricas em oxigênio (Krammer 2000).

Espécie pioneira para o estado do Paraná.

Ocorrência nas amostras: UPCB 63367.

\section{Pinnularia sp.}

Fig. 25

Valvas lineares com margens paralelas; extremidades valvares subcapitadas; esterno da rafe lanceolado, de modo que um lado é mais estreito que o outro; área central lateralmente expandida, formando uma fáscia; rafe filiforme levemente sinuosa; extremidades proximais fletidas para o mesmo lado; extremidades terminais em forma de foice; estrias alveoladas radiadas, convergentes em direção as extremidades. Compr.: $59,5 \mu \mathrm{m}$; lar. 8,63 $\mu \mathrm{m} ; 11$ estrias em $10 \mu \mathrm{m}$.

$\mathrm{O}$ único exemplar encontrado assemelhou-se a Pinnularia subgibba var. lanceolata Gaiser \& Johansen, porém esta difere pelas valvas mais lanceoladas e extremidades rostradas mais afiladas (Gaiser \& Johansen 2000). A variedade undulata Krammer possui valvas lineares com ápices mais arredondados, em ambas as variedades o esterno é amplo.

Ocorrência nas amostras: UPCB 63363.

Família NAVICULACEAE

Navicula kuseliana Lange-Bertalot \& Rumrich, Iconogr.

Diatomol. 9: 161, pl. 43, fig. 1-8, 2000.

Fig. 26

Valvas lanceoladas; extremidades sub-rostradas; esterno da rafe linear, estreito; área central estreita e rômbica com uma estria mais curta que as demais; rafe reta, filiforme; estrias radiais na porção mediana da valva e paralelas nas extremidades. Compr.: 44,0-54,4 $\mu \mathrm{m}$; larg.: 8,8-10,4 $\mu \mathrm{m}$; 13-15 estrias em $10 \mu \mathrm{m}$.

Espécie associada a ambientes de águas eutróficas (Rumrich et al. 2000).

Espécie pioneira para o estado do Paraná.

Ocorrência nas amostras: UPCB 63363, UPCB 63365, UPCB 63367 e UPCB 63368.

Navicula viridulacalcis var. neomundana Lange-Bertalot \& Rumrich, Iconogr. Diatomol. 9: 175; pl. 37, fig. 5-8, pl. 38, fig. 1-4, 2000.

Fig. 27

Valvas lineares; extremidades cuneadas; esterno da rafe linear, estreito; área central arredondada com estrias encurtadas regularmente; rafe reta, filiforme com extremidades distais curvadas; estrias radiais na porção mediana da valva e paralelas nas extremidades. Compr.: 53,7-60 $\mu \mathrm{m}$; larg.: 10,2-11,3 $\mu \mathrm{m}$; 9-10 estrias em $10 \mu \mathrm{m}$.

Não foram encontrados dados ecológicos na literatura.

Espécie pioneira para o estado do Paraná.

Ocorrência nas amostras: UPCB 63363.

Navicula wildii Lange-Bertalot; Biblioth. Diatomol. 27(2/14): 141-142, fig. 46 (9-13), 1993.

Fig. 28

Valvas lanceoladas; extremidades arredondadas a levemente rostrada; esterno da rafe linear, estreito; área 
central lanceolada, com a estria mediana circundada por duas estrias menores, em ambas as margens; rafe filiforme, reta; estrias radiais se apresentando paralelas em direção as extremidades. Compr.: 44,0-57,6 $\mu \mathrm{m}$; larg.: 6,4-9,6 $\mu \mathrm{m}$; 13-15 estrias em $10 \mu \mathrm{m}$.

Espécie de água doce e salobra, oligotrófica e de $\mathrm{pH}$ alcalino (van Dam et al. 1994, Hofmann 1994).

Espécie pioneira para o estado do Paraná.

Ocorrência nas amostras: UPCB 63363, UPCB 63365 e UPCB 63368.

\section{Eolimna sp.}

Fig. 29-30

Valvas lanceoladas; extremidades amplamente rostradas; esterno da rafe linear, estreito; área central reduzida, delimitada pelo encurtamento das estrias medianas; rafe filiforme, reta; estrias radiadas em toda superfície valvar. Compr.: 10,0-15,2 $\mu \mathrm{m}$; larg.: 4,0-4,78 $\mu \mathrm{m}$, estrias inconspícuas.

Diferencia-se de Eolimna verecundaeformis (Manguin) Moser, Lange-Bertalot \& Metzeltin, por esta apresentar estrias medianas mais curtas, região central retangular e ápice rostrado pouco destacado do corpo valvar (Moser et al., 1998).

Ocorrência nas amostras: UPCB 63363 e UPCB 63367.

Família STAURONEIDACEAE

Stauroneis microbtusa Reichardt, Iconogr. Diatomol. 13: 442, pl. 9, fig. 7-12, pl. 10, fig. 5, 2004.

Fig. 31-32

Valvas linear-elipticas; extremidades arredondadas; esterno da rafe linear, estreito; rafe reta, com extremidade proximal expandida como poro; área central ampla, alcançando as margens valvares; estrias radiadas ao longo da margem valvar. Compr.: 17,8-20,0 $\mu \mathrm{m}$; larg.: 4,8-5,6 $\mu \mathrm{m}$, estrias de difícil contagem.

Não foram encontrados dados ecológicos na literatura.

Espécie pioneira para o estado do Paraná.

Ocorrência nas amostras: UPCB 63367.

\section{Stauroneis sp.}

Fig. 33

Valvas lanceoladas; extremidades capitadas; esterno da rafe mais largo em direção as extremidades; estauro
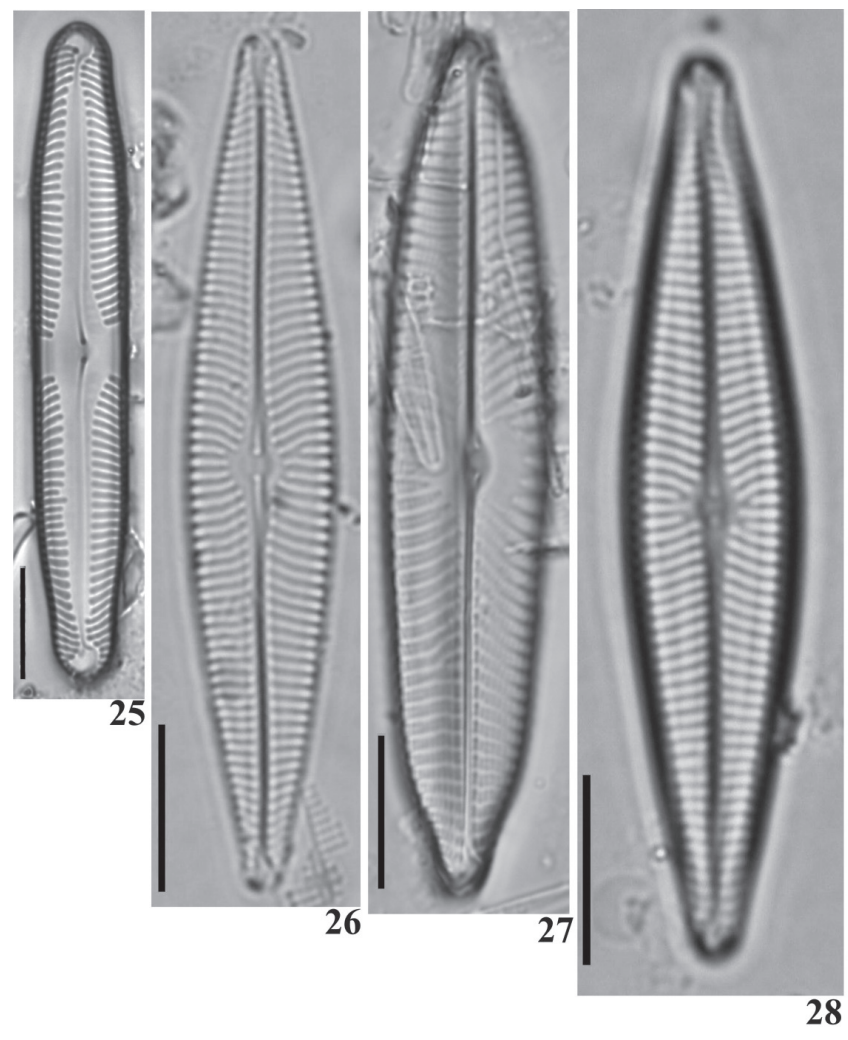
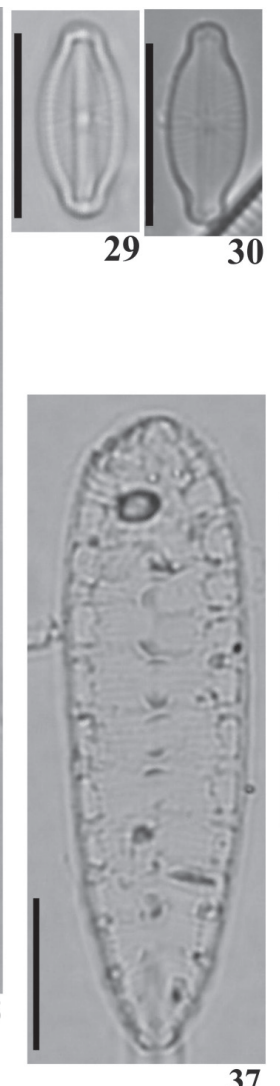
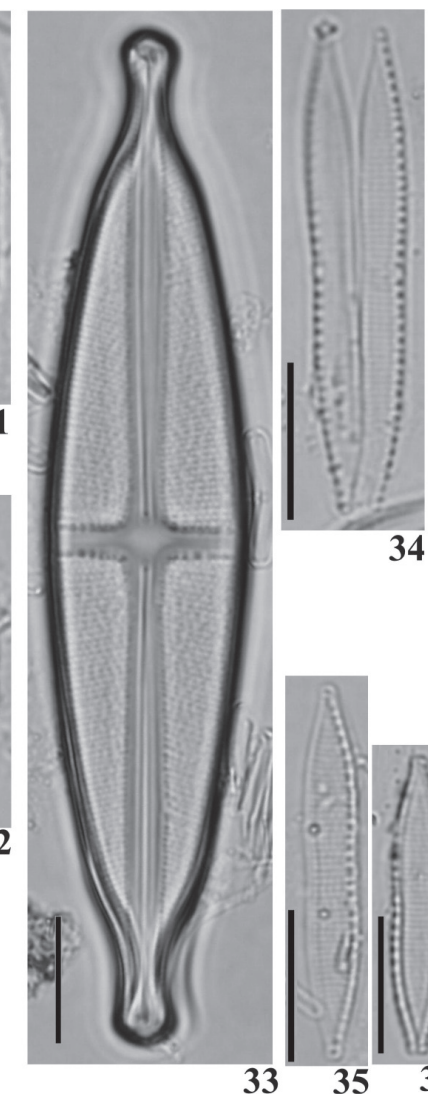

34

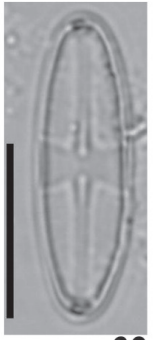

32

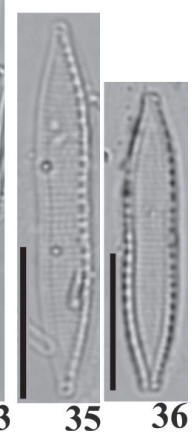

Figuras 25-37. Diatomáceas perifíticas do reservatório eutrófico do Iraí. 25 Pinnularia sp.; 26 Navicula kuseliana Lange-Bertalot \& Rumrich; 27 Navicula viridulacalcis var. neomundana Lange-Bertalot \& Rumrich; 28 Navicula wildii Lange-Bertalot; 29-30 Eolimna sp.; 31-32 Stauroneis microbtusa Reichardt; 33 Stauroneis sp.; 34-36 Nitzschia sp.; 37 Surirella sp. Escala: $10 \mu \mathrm{m}$. 

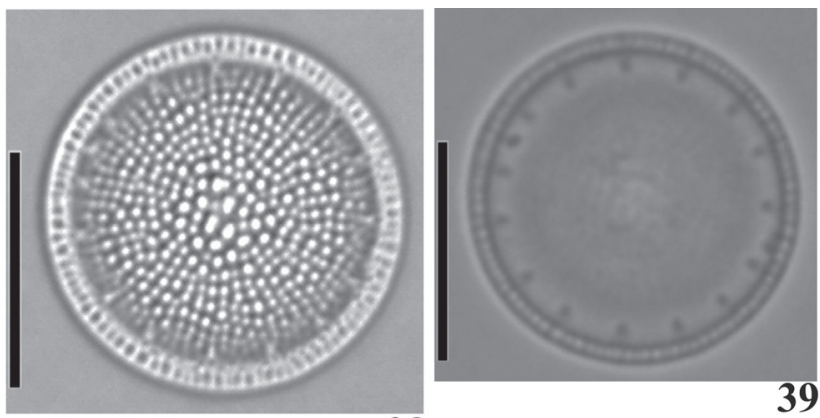

38

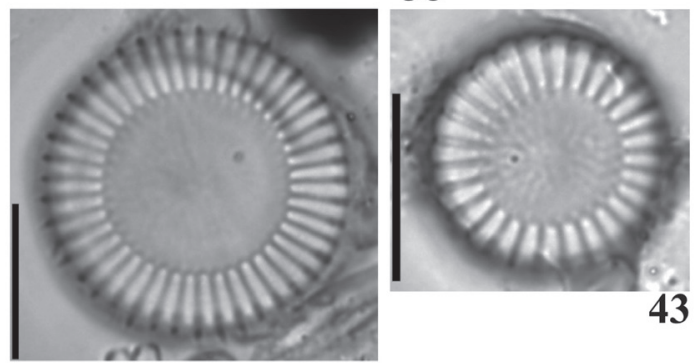

42

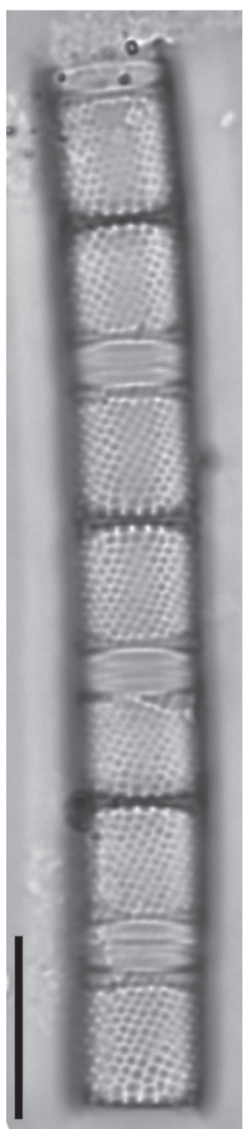

47

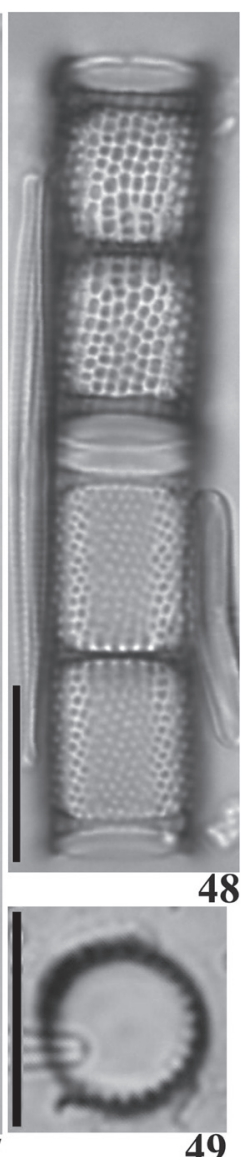

49

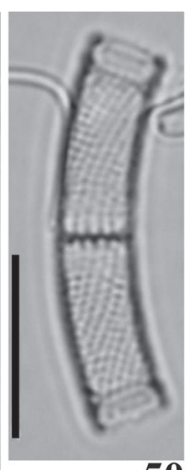

50

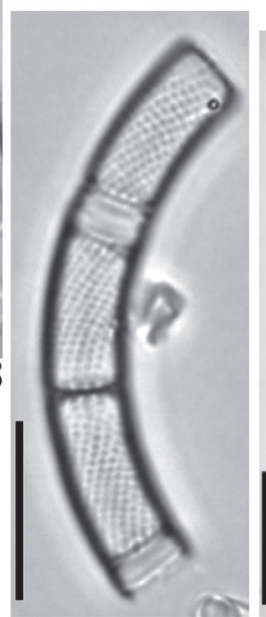

51
43

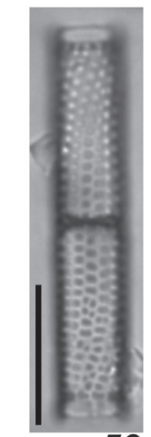

52
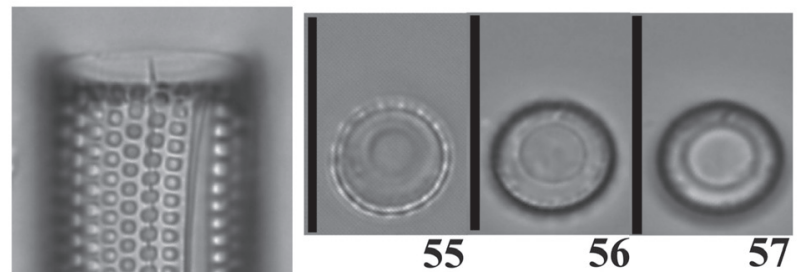

:

:이용

:08go :

:009 :

:
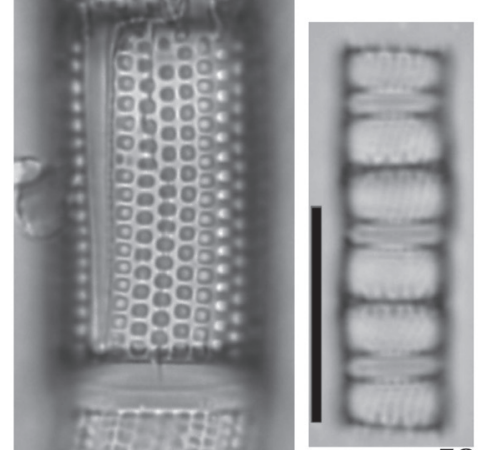

58

59

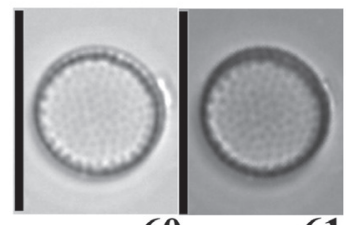

60

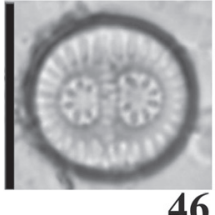

46

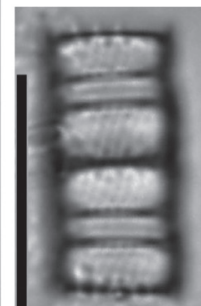

5ysth

s.7.

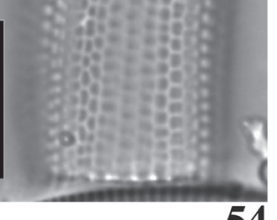

54
61

Figuras 38-61. Diatomáceas perifíticas do reservatório eutrófico do Iraí.38-41 Thalassiosira rudis Tremarin, Ludwig, Beker \& Torgan; 42-43 Cyclotella meneghiniana Kützing; 44-46 Discostella stelligera (Cleve \& Grunow) Houk \& Klee; $47-48$ Aulacoseira ambigua var. ambigua f. ambigua (Grunow) Simonsen (vista pleural); 49 Aulacoseira ambigua var. ambigua f. ambigua (Grunow) Simonsen (vista valvar); 50-51 Aulacoseira ambigua var. ambigua f. spiralis (Skuja) Ludwig \& ValenteMoreira; 52-54 Aulacoseira granulata var. granulata (Ehrenberg) Simonsen; 55-57 Aulacoseira pusilla (Meister) Tuji \& Houki (vista valvar); 58-59 Aulacoseira pusilla (Meister) Tuji \& Houki (vista pleural); 60-61 Aulacoseira tenella (Nygaard) Simonsen. Escala: $10 \mu \mathrm{m}$. 


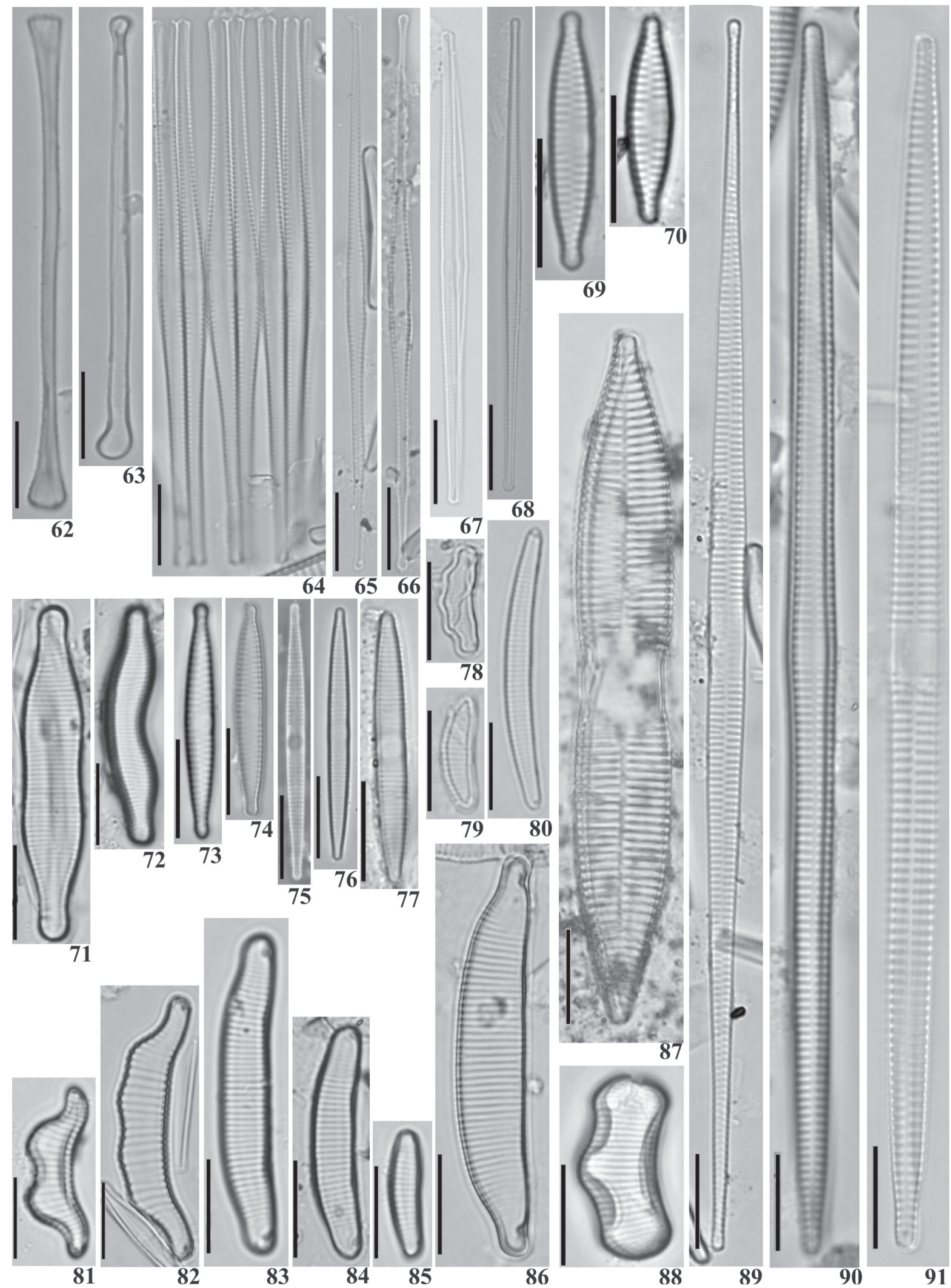

Figuras 62-91. Diatomáceas perifíticas do reservatório eutrófico do Iraí.62-63 Asterionella formosa Hassal; 64-66 Fragilaria crotonensis Kitton; 67-68 Fragilaria gracilis Østrup; 69-70 Fragilaria vaucheriae (Kützing) Petersen; 71-72 Fragilaria javanica Hustedt; 73-74 Fragilaria vaucheriae var. capitellata (Grunow) Patrick; 75-77 Fragilaria rumpens (Kützing) Carlson; 78 Eunotia muscicola var. tridentula Nörpel \& Lange-Bertalot; 79 Eunotia subarcuatoides Alles, Nörpel-Schempo \& Lange-Bertalot; 80 Eunotia bilunaris (Ehrenberg) Mills; 81-82 Eunotia camelus Ehrenberg; 83-84 Eunotia minor (Kützing) Grunow; 85 Eunotia rhomboidea Hustedt; 86 Eunotia pirla Carter \& Flower; 87 Synedra goulardii Brébisson ex Cleve \& Grunow; 88 Eunotia rabenhorstii Cleve \& Grunow; 89 Ulnaria delicatissima (Wm. Smith) Aboal \& Silva; 90-91 Ulnaria ulna (Nitzsch) Compère. Escala: $10 \mu \mathrm{m}$. 

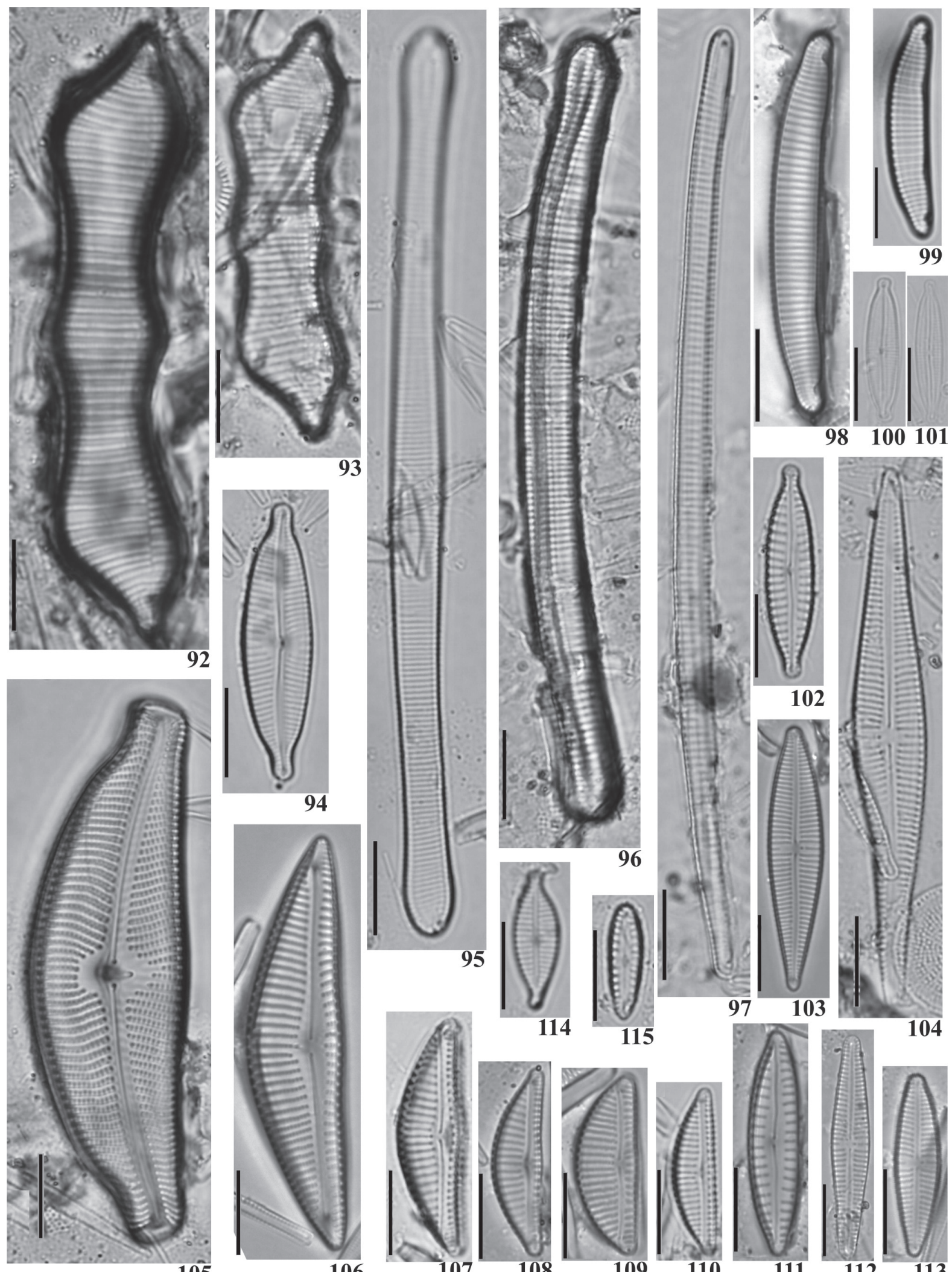

105
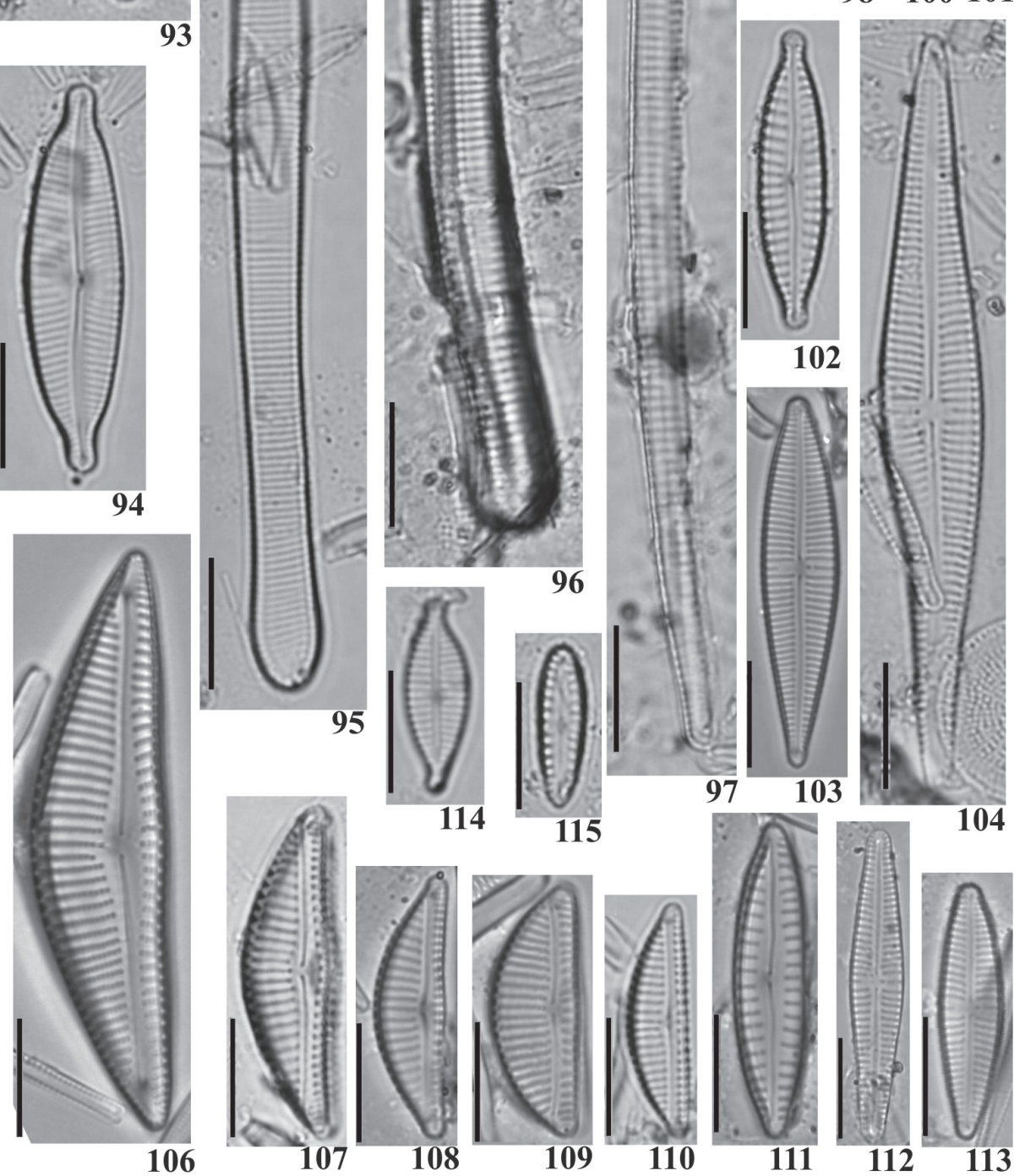

Figuras 92-115. Diatomáceas perifíticas do reservatório eutrófico do Iraí.92-93 Eunotia didyma Grunow; 94 Cymbopleura naviculiformis (Auerswald) Krammer; 95 Eunotia flexuosa Brébisson ex. Kützing; 96 Eunotia major (Wm. Smith) Rabenhorst; 97 Eunotia naegelii Migula in Thomé; 98 -99 Eunotia sudetica O. Müller; 100 -101 Encyonopsis microcephala (Grunow) Krammer; 102 Encyonopsis schubartii (Hustedt) Krammer; 103-104 Gomphonema gracile Ehrenberg; 105 Cymbella tumida (Brébisson) Van Heurck; 106 Encyonema neomesianum Krammer; 107-108 Encyonema minutum (Hilse) Mann; 109-110 Encyonema silesiacum (Bleisch) Mann; 111 Encyonopsis difficilis (Krasske) Krammer; 112-113 Gomphonema parvulum (Kützing) Kützing; 114 Gomphonema lagenula Kützing; 115 Gophonema pumilum (Grunow) Reichardt \& Lange-Bertalot. Escala: $10 \mu \mathrm{m}$. 

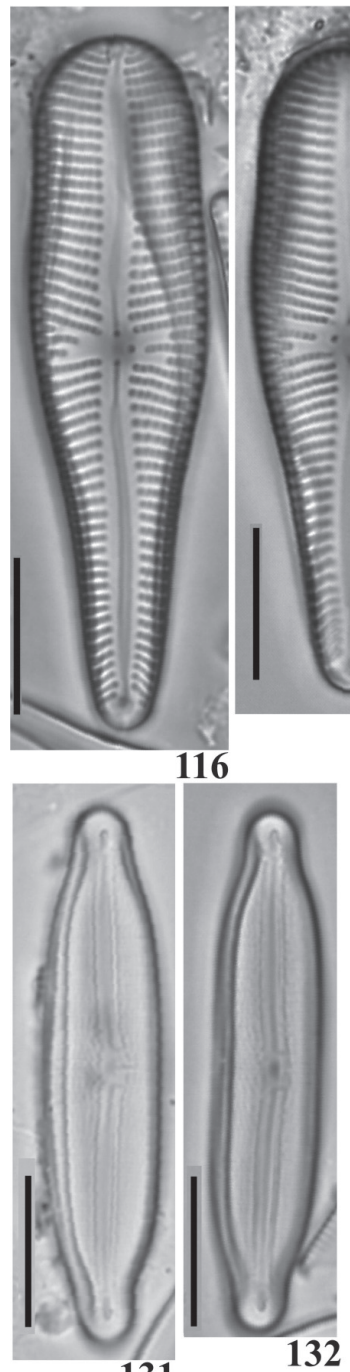

131
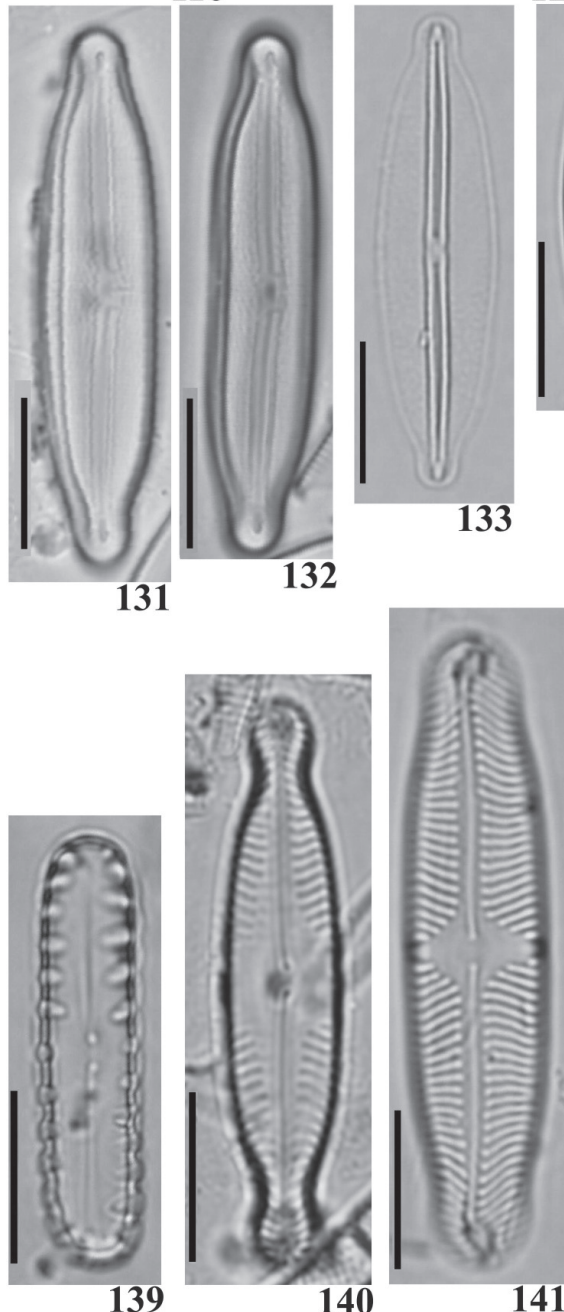

139

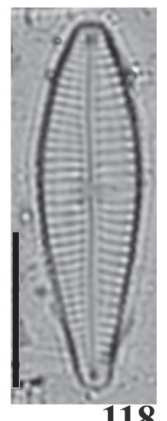

118

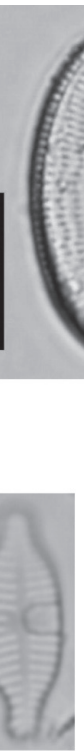

124

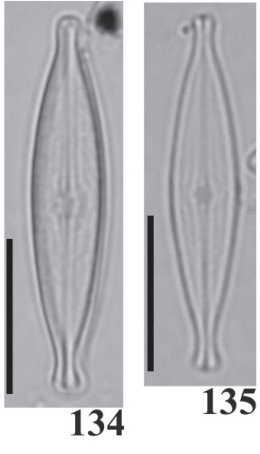

126
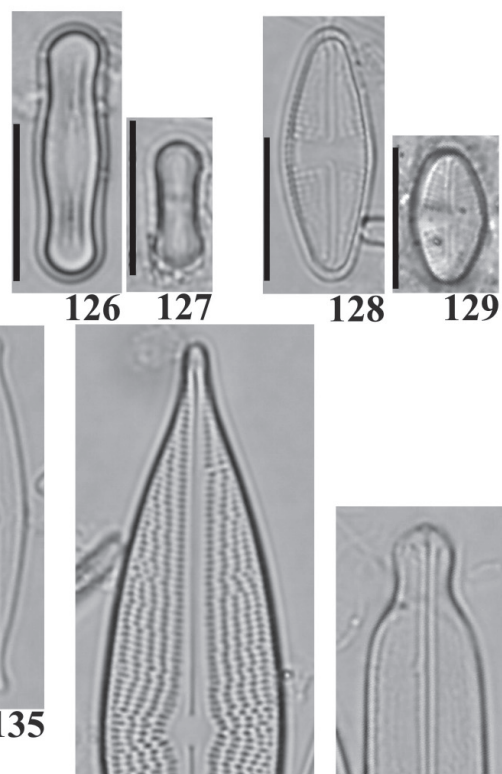

128

129
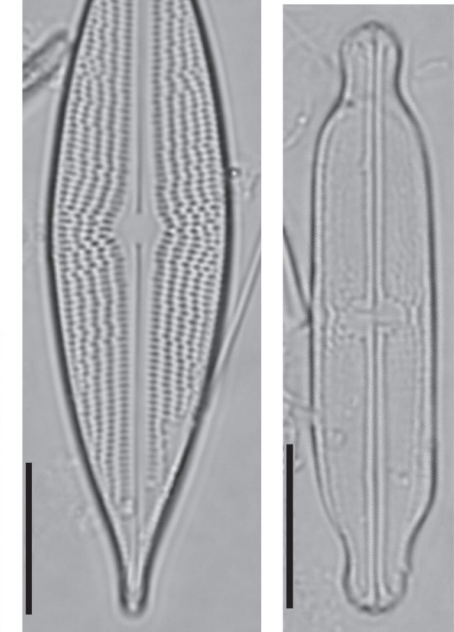

137

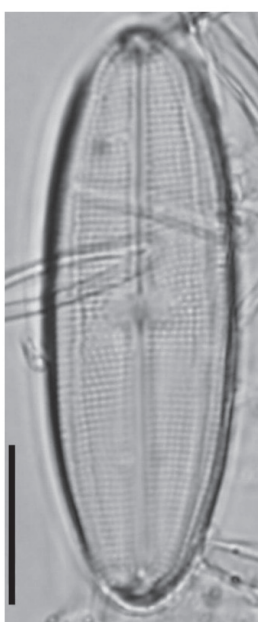

138
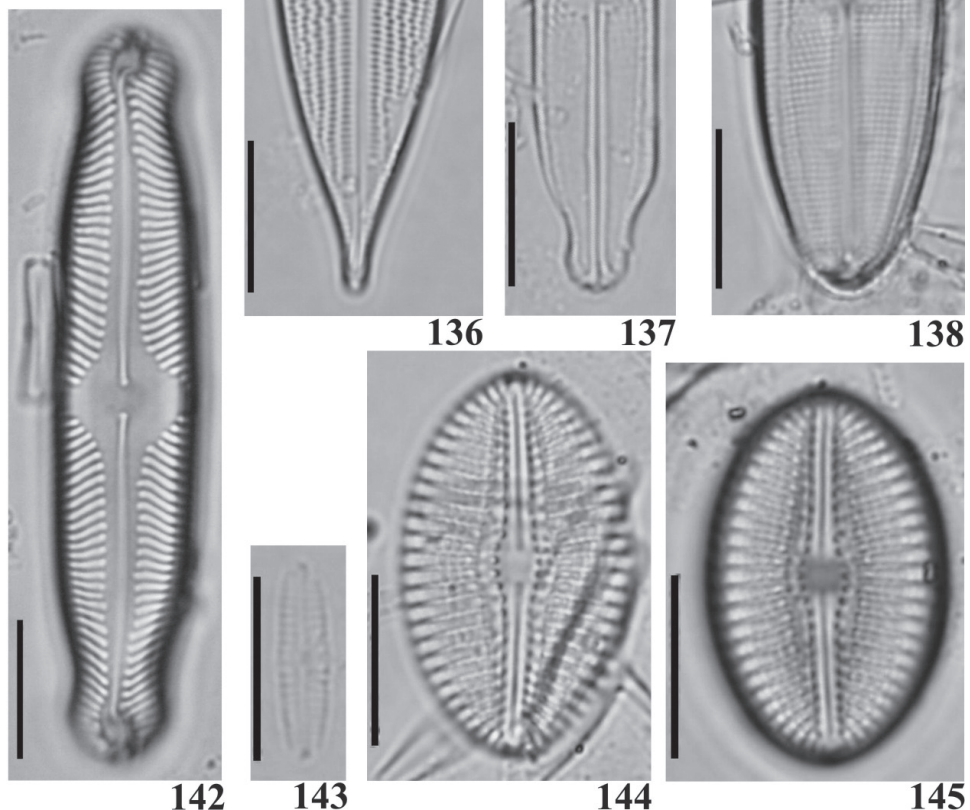

Figuras 116-145. Diatomáceas perifíticas do reservatório eutrófico do Iraí.116-117 Gomphonema laticollum Reichardt; 118 Gomphonema pseudoaugur Lange-Bertalot; 119 Cocconeis placentula var. lineata (Ehrenberg) Van Heurck; 120-123 Achnanthidium minutissimum (Kützing) Czarnecki; $124-125$ Planothidium biporomum (Hohn \& Hellerman) Lange-Bertalot; 126-127 Diadesmis contenta (Grunow ex Van Heurck) Mann; 128-129 Luticola mutica (Kützing) Mann; 130 Frustulia crassinervia (Brébisson) Costa; 131-132 Frustulia neomundana Lange-Bertalot \& Rumrich; 133 Frustulia saxonica Rabenhorst; 134-135 Brachysira neoexilis Lange-Bertalot; 136 Brachysira rostrata (Krasske) Metzeltin \& Lange-Bertalot; 137 Neidium affine (Ehrenberg) Pfitzer; 138 Neidium ampliatum (Ehrenberg) Krammer; 139 Pinnularia borealis var. rectangularis Carlson; 140 Pinnularia brauniana (Grunow) Mills; 141-142 Pinnularia divergens var. media Krammer; 143 Chamaepinnularia mediocris (Krasske) Lange-Bertalot; 144 Diploneis ovalis (Hilse) Cleve; 145 Diploneis subovalis Cleve. Escala: $10 \mu \mathrm{m}$. 

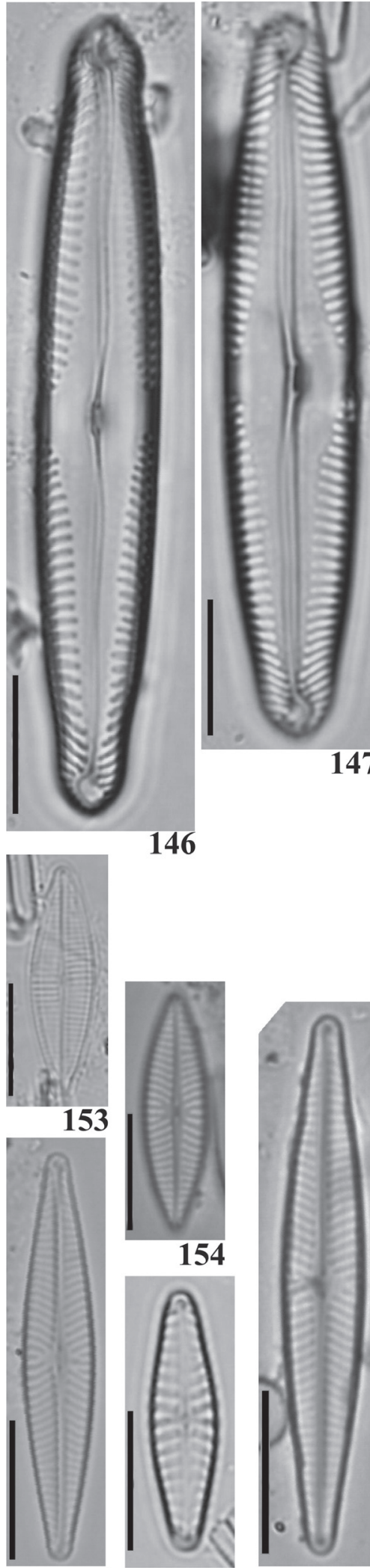

155

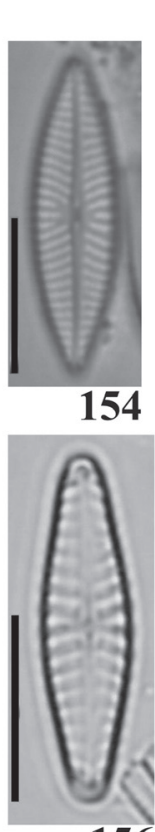

156

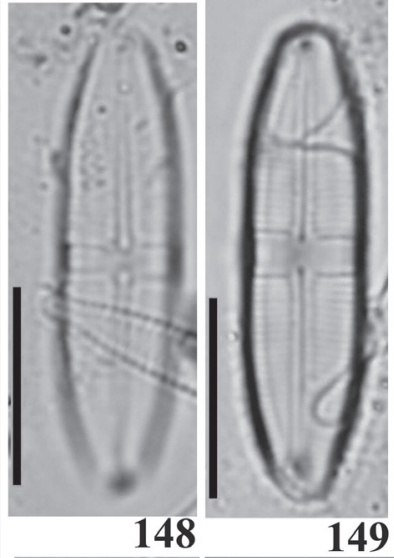

149
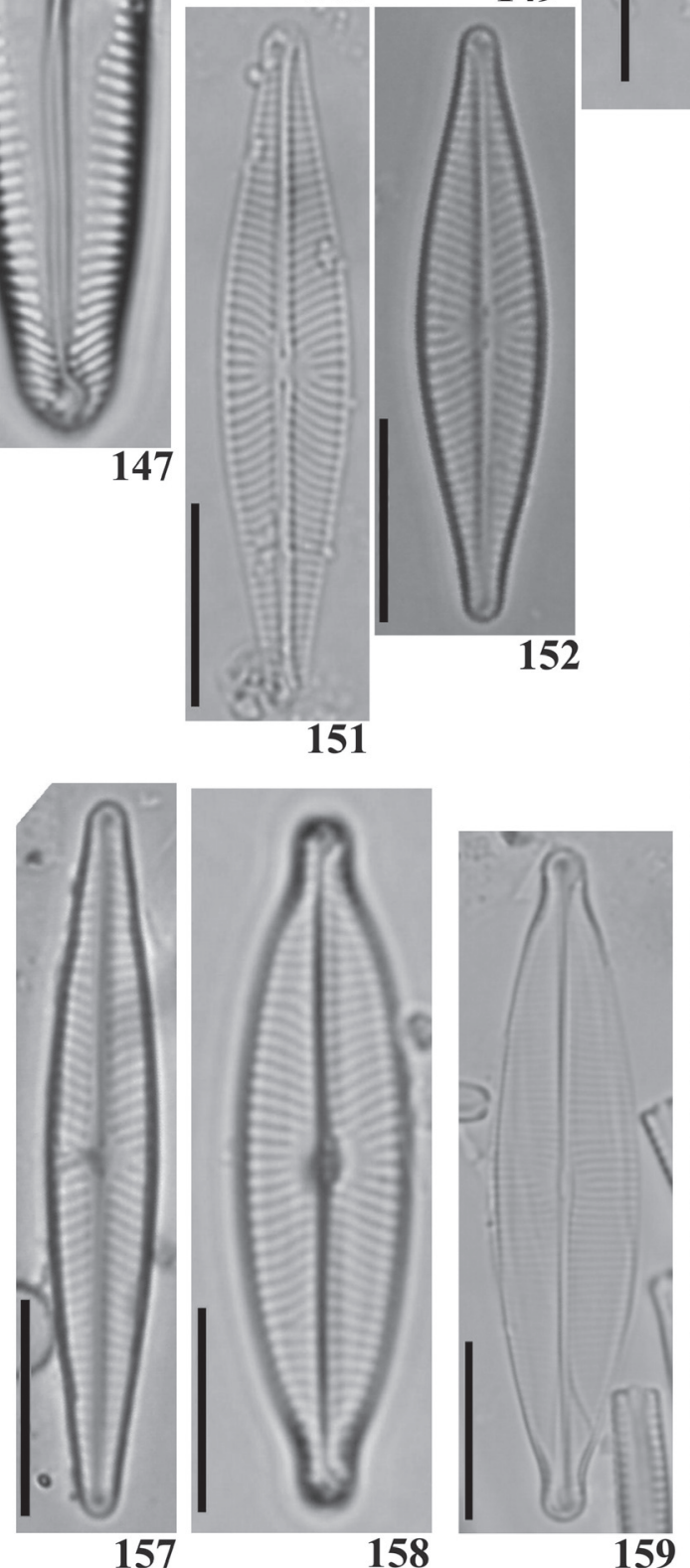

159
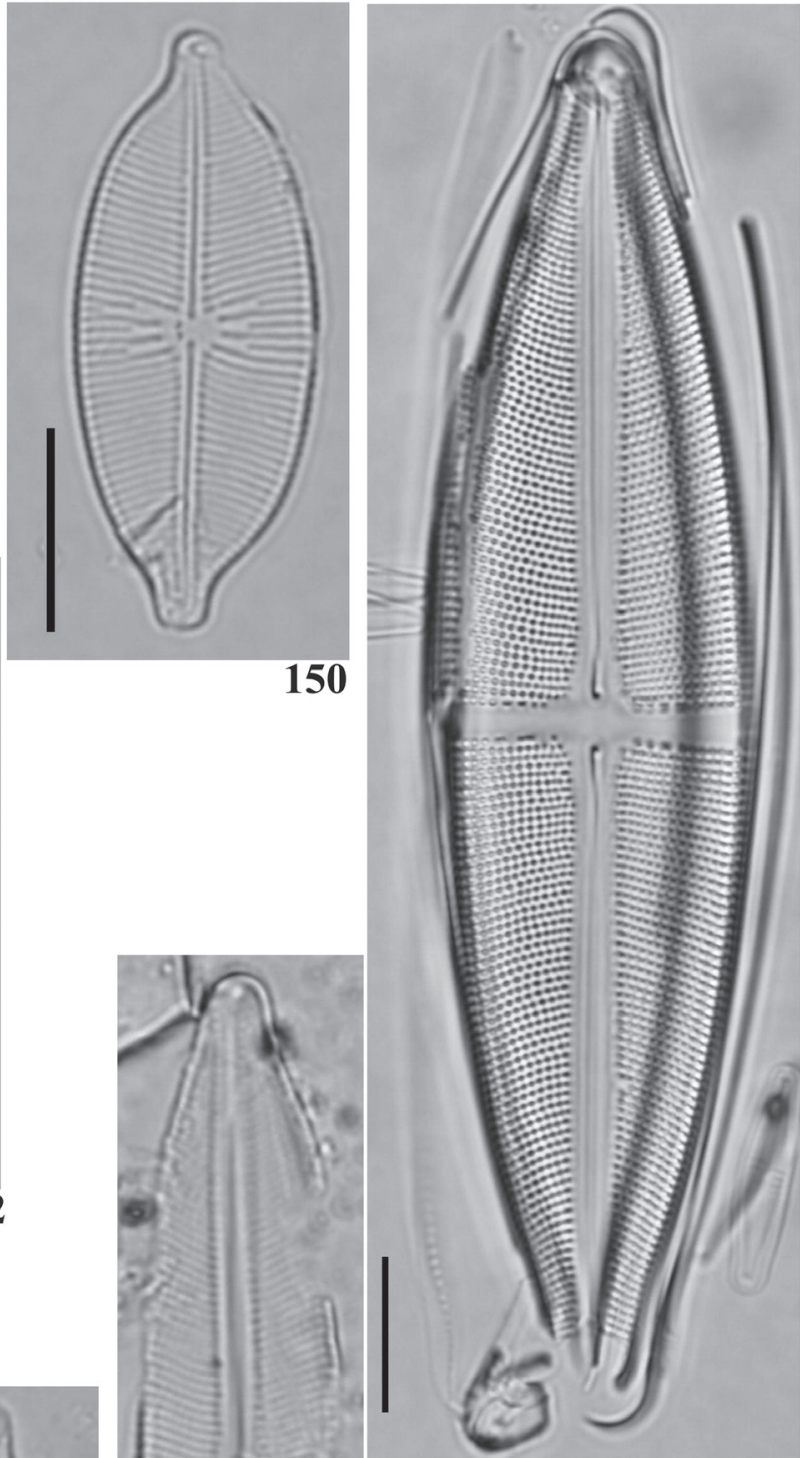

161
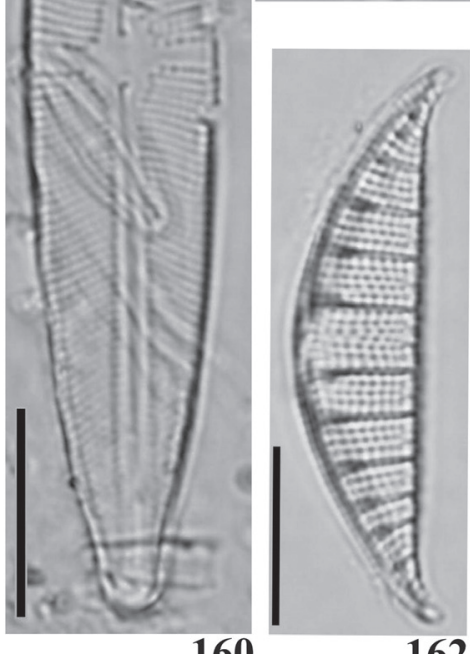

162

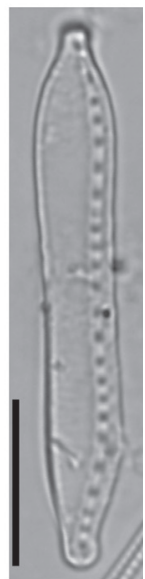

163

Figuras 146-163. Diatomáceas perifíticas do reservatório eutrófico do Iraí.146-147 Pinnularia gibba Ehrenberg; 148-149 Caloneis bacillum (Grunow) Cleve; 150 Navicula clementis var. linearis Brander ex Hustedt; 151-152 Navicula cryptocephala Kützing; 153 Chamaepinnularia mediocris (Krasske) Lange-Bertalot; 154-155 Navicula cryptotenella Lange-Bertalot; 156 Hippondonta avittata (Cholnoky) Lange-Bertalot; 157 Navicula leptostriata Jorgensen; 158 Navicula rostellata Kützing; 159 Craticula halophila (Grunow ex Van Heurck) Mann; 160 Stauroneis gracilis Ehrenberg; 161 Stauroneis phoenicenteron (Nitzsch) Ehrenberg; 162 Rhopalodia gibberula var. vanheurckii O. Müller; 163 Hantzchia amphioxys (Ehrenberg) Grunow. Escala: $10 \mu \mathrm{m}$. 

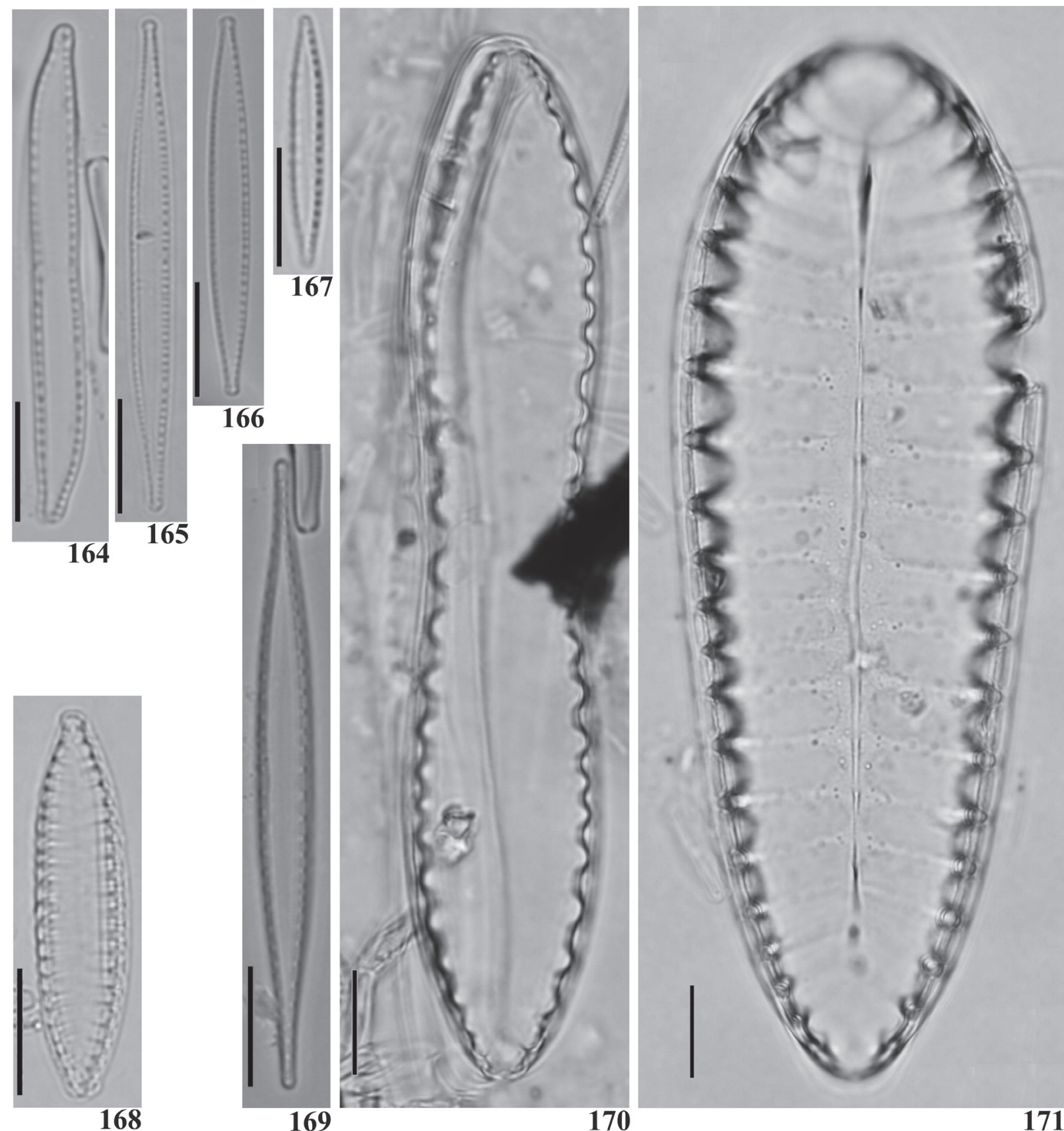

168

169

170

171

Figuras 164-171. Diatomáceas perifíticas do reservatório eutrófico do Iraí.164 Nitzschia clausii Hantzsch; 165 Nitzschia gracilis Hantzsch; 166 Nitzschia palea (Kützing) Wm. Smith; 167 Nitzschia perminuta (Grunow) Peragallo; 168 Surirella angusta Kützing; 169 Stenopterobia delicatissima (Lewis) Van Heurck; 170 Surirella linearis Smith var. constricta Grunow; 171 Surirella splendida (Ehrenberg) Kützing. Escala: $10 \mu \mathrm{m}$.

linear presente; rafe filiforme, reta com extremidades simples; estrias delicadas, radiadas; aréolas inconspícuas. Compr.: 55,2-83,2 $\mu \mathrm{m}$; larg.: 9,6-16,0 $\mu \mathrm{m}, 18-22$ estrias em $10 \mu \mathrm{m}$.

Assemelha-se a Stauroneis gracilior (Rabenhorst) Reichardt, porém apresenta medidas maiores e esterno da rafe mais largo. Semelhante ao Stauroneis sp. apresentado por Metzeltin \& Lange-Bertalot (2007, p. 504, fig. 3).

Ocorrência nas amostras: UPCB 63367.

\section{Família BACILLARIACEAE Nitzschia sp.}

Fig. 34-36

Valvas lineares; extremidades subcapitadas a rostradas; fíbulas mais espaçadas no centro da valva e eqüidistantes em direção as extremidades; rafe marginal; estrias inconspícuas. Compr.: 28,0-32,0 $\mu \mathrm{m}$; larg.: 3,2 $\mu \mathrm{m}$; 22-24 estrias em 10 $\mu \mathrm{m} ; 9-13$ fíbulas em $10 \mu \mathrm{m}$.

Diferencia-se de Nitzschia hantzschiana Rabenhorst por apresentar maior densidade de fíbulas, e por estas es- 
tarem dispostas mais próximas umas das outras do que as observadas nos exemplares de $N$. hantzschiana (Krammer \& Lange-Bertalot 1988, pl. 73, figs. 9-18).

Ocorrência nas amostras: UPCB 63363, UPCB 63364, UPCB 63365, UPCB 63367, UPCB 63368, UPCB 63369 e UPCB 63370.

\section{Família SURIRELLACEAE Surirella sp.}

Fig. 37

Valvas heteropolares, linear ovada; extremidade superior arredondada; extremidade inferior cuneada arredondada; esterno inconspícuo; projeções aliformes paralelas; estrias delicadas. Compr.: 42,1 $\mu \mathrm{m}$; larg.: 11,3 $\mu \mathrm{m}, 16$ estrias em $10 \mu \mathrm{m} ; 3$ processos aliformes em $10 \mu \mathrm{m}$.

Ocorrência nas amostras: UPCB 63369.

\section{Considerações sobre as espécies predominantes}

Dados ecológicos sobre 73 espécies foram pesquisados e incluídos, sendo que $35 \%$ foram classificadas como presentes em ambientes eutróficos, $18 \%$ em águas mesotróficas e $27 \%$ em sistemas oligotróficos. Espécies classificadas como indiferentes e tolerantes foram $19 \%$.

De maneira geral, as maiores densidades da comunidade de diatomáceas perifíticas ocorreram na estação chuvosa. A espécie mais representativa nas amostras do reservatório foi Achnanthidium minutissimum que se apresentou dominante em todas as estações, sempre em quantidade igual ou superior a 74\% do total contado. Espécies consideradas abundantes foram Fragilaria rumpens, com valor de 5\% em novembro/2007 e maio/2008 e $7 \%$ em agosto/2008, Fragilaria vaucheriae var. capitellata, com 4\% em novembro/2007 e agosto/2008) e Gomphonema gracile, com 5\% em agosto de 2008.

Baseando-se em literatura (van Dam et al., 1994; Hoffman, 1994; Potapova \& Charles, 2007), a comunidade de diatomáceas do reservatório do Iraí esteve composta por espécies desde sensíveis ao processo de eutrofização até espécies tolerantes (Tabela 2). Round et al. (1990) ressaltaram que A. minutissimum apresenta amplo espectro de tolerância às mudanças ambientais podendo ser encontrada em ambientes pobres em nutrientes, mas também em ambientes extremamente eutrofizados. Entretanto, Lobo et al. (2002) classificara-na como característica de ambiente fortemente poluído ( $\alpha$-polissapróbico).

Fragilaria rumpens foi enquadrada como espécie tolerante a ambientes em processo de eutrofização (mesoeutróficos) por Stenger-Kovács et al. (2007) e Lobo et al. (2002). G. gracile foi considerada como de ambientes mesossapróbicos por Lobo et al. (2002) e eutróficos por Hofmann (1994) e F. vaucheriae var. capitellata classificada como de ambientes eutróficos (van Dam 1994).

Táxons que apresentaram mais de $1 \%$ de representatividade, mas que não foram considerados abundantes no sistema foram Aulacoseira ambigua, Aulacoseira granulata,
Aulacoseira pusilla, Brachysira neoexilis, Cyclotella meneghiniana, Cymbella tumida, Discostella stelligera, Fragilaria crotonensis, Fragilaria gracilis, Fragilaria vaucheriae, Fragilaria vaucheriae var. capitellata, Gomphonema lagenula, Gomphonema laticollum, Gomphonema parvulum, Navicula cryptotenella, Nitzschia palea, Ulnaria delicatissima e Ulnaria ulna. Dentre esses $B$. neoexilis, D. stelligera, F. gracilis e U. delicatissima (van Dam et al. 1994, Lange-Bertalot 2004) foram classificados como de ambientes oligo-mesotróficos, sendo os demais categorizados como de ambientes mesoeutróficos e indiferentes a tolerantes ao processo de eutrofização (van Dam et al. 1994, Hofmann 1994, Krammer 2000).

\section{Agradecimentos}

Ao Conselho Nacional de Desenvolvimento Científico e Tecnológico (CNPq) pela concessão da bolsa de mestrado (AMS), da bolsa de produtividade (TAVL) e do apoio financeiro para a realização do projeto (CTHidro555397/2006-8). Ao IAP e SANEPAR por viabilizar a realização das coletas.

\section{Referências Bibliográficas}

Ács, É.; Reskóné, N.M.; Szabó, K.; Taba, G. \& Kiss, K.T. 2005. Application of epiphytic diatoms in water quality monitoring of lake VelenceRecommendations and Assignments. Acta Botanica Hungarica 47(3-4): 211-223.

Alles, E.; Nörpel-Schempp, M. \& Lange-Bertalot, H. 1991. Zur Systematik und Ökologie charakteristischer Eunotia-Arten (Bacillariophyceae) in elektrolyt-armen Bachoberläufen. Nova Hedwigia 53(1-2): 171-213.

Barber, H. G. \& Haworth, E.Y. 1981. A guide to the morphology of the diatom frustule. Cumbria, Freshwater Biological Association.

Bicudo, C.E.M. \& Menezes, M. 2006. Gêneros de algas de águas continentais do Brasil. São Carlos, Rima.

Carneiro, L.A. 2007. Fragilariophyceae (Ocrhrophyta) de águas doces do estado de São Paulo: Levantamento florístico. 196f. Tese (Doutorado em Biodiversidade Vegetal e Meio Ambiente)-Instituto de Botânica da Secretaria de Estado do Meio Ambiente, São Paulo.

Carlson, R.E. 1977. A trophic state index for lakes. Limnology and Oceanography 22(2): 361-369.

Carter, J.R. \& Flower, R.J. 1988. A new species of Eunotia, E. pirla sp. nov., from Woolmer pond, an acid pool in the southeast of England. Diatom Research 3: 1-8.

Cetto, J.M.; Leandrini, J.A.; Felisberto, S.A. \& Rodrigues, L. 2004. Comunidade de algas perifíticas no reservatório de Irai, Estado do Paraná, Brasil. Acta Scientiarum. Biological Sciences Maringá 26(1): 1-7.

Cox, E.J. 1987. Placoneis Mereschkowsky: the re-evaluation of a diatom genus originally characterized by its chloroplast type. Diatom Research 2(2): 145-157.

Czarnecki, D.B. 1994. The freshwater diatom culture collection at Loras College, Dubuque, Iowa. Pp. 155-174. In: Kociolek, J.P. (ed.). Proceedings of the 11th International Diatom Symposium, San Francisco 1990. Memoirs of the California Academy of Sciences, n. 17.

Dela-Cruz, J.; Pritchard, T.; Gordon, G. \& Ajani, P. 2006. The use of periphytic diatoms as a means of assessing impacts of point source inorganic nutrient pollution in south-eastern Australia. Freshwater biology 51: 951-972.

Descy, J.P. 1979. A new approach to water quality estimation using diatom. Nova Hedwigia 64: 305-323.

Dinnerstein, E.; Olson, D.M.; Graham, D.J.; Webstar, A.L. \& Primm, A.S. 1995. A conservation assessment of the terrestrial ecoregions of Latin America and the Caribbean. Washington, The World Bank.

Dixit, S.S. \& Smol, J.P. 1995. Diatom evidence of past water quality changes in Adirondack Seepage Lakes (New York, U.S.A.). Diatom Research 10(1): 113-129.

Ferrari, F. \& Ludwig, T.A.V. 2007. Coscinodiscophyceae, Fragilariophyceae e Bacillariophyceae (Achnanthales) dos rios Ivaí, São João e dos Patos, bacia hidrográfico do rio Ivaí, município de Prudentópolis, PR, Brasil. Acta Botanica Brasilica 21(2): 421-441. 
Fonseca, I.A. \& Rodrigues, L. 2005. Comunidade de algas perifíticas em distintos ambientes da planície de inundação do alto rio Paraná. Acta Scientiarum. Biological Sciences Maringá 27(1): 21-28.

Gaiser, E.E. \& Johansen, J. 2000. Freshwater diatoms from Carolina Bays and other isolatedt Wetlands on the Atlantic Coastal Plain of South Carolina, U.S.A., with descriptions of seven taxa new to science. Diatom Research 15: 75-130.

Góméz, N. \& Licursi, M. 2001. The Pampean Diatom Index (IDP) for assessment of rivers and streams in Argentina. Aquatic Ecology 5: 173-181.

Hånkansson, S. 1993. Numerical methods for the inference of $\mathrm{pH}$ variations in mesotrophic and eutrophic lakes in Southern Sweden-A progress report. Diatom Research 8(2): 349-370.

Hofmann, G. 1994. Aufwuchs-Diatomeen in Seen und ihre Eignung als Indikatoren der Trophie. Bibliotheca Diatomologica 30: 1-241.

Houk, V. \& Klee, R. 2004. The Stelligeroid taxa of the genus Cyclotella (Kützing) Brébisson (Bacillariophyceae) and their transfer into the new genus Discostella gen. nov. Diatom Research 19(2): 203-228.

Hustedt, F. Die Kieselalgen. 1927-1930. In: Rabenhorst, L. KryptogamenFlora. Leipzig: Akademische Verlagsgesellschaft, 7(1): 920.

Hustedt, F. Die Kieselalgen. 1927-1966. Die Kieselalgen. In: Rabenhorst, L. Kryptogamen-Flora. Leipzig: Akademische Verlagsgesellschaft 7(1-3): 1-920, 1-845, 1-816.

Hustedt, F. 1961-1966. Die Kieselalgen. In: Rabenhorst, L. KryptogamenFlora. Leipzig: Akademische Verlagsgesellschaft, 7(3): 816.

Ivanov, P. \& Kirilova, E. 2004. Benthic diatoms assemblages from different substrates of the Iskar river, Bulgária. Pp. 107-124. In: Witkowski, A. (ed.). Proceeding of the Eighteenth International Diatom Symposium, Miedzyzdroje

Kelly, M.G.; Whitton, B.A. 1995. The trophic diatom index: a new index for monitoring eutrophication in rivers. Journal of Applied Phycology 7: 433-444.

Kelly, M.G.; Cazaubon, A.; Coring, E.; Dell'Uomo, A.; Ector, L.; Goldsmith, B.; Guasch, H.; Hürlimann, J.; Jarlman, A.; Kawecka, B.; Kwandrans, J.; Laugaste, R.; LindstrØm, E.A.; Leitao, M.; Marvan, P.; Padisák, J.; Pipp, E.; Prygiel, J.; Rott, E.; Sabater, S.; van Dam, H. \& Vizinet, J. 1998. Recommendations for the routine samping of diatoms for water quality assessments in Europe. Journal of Applied Phycology 10: 215-224.

Krammer, K. 2000. The genus Pinnularia. Diatoms of Europe 1: 1-703.

Krammer, K. 2003. Cymbopleura, Delicata, Navicymbula, Gomphocymbellopsis, Afrocymbella. Diatoms of Europe 4: 529

Krammer, K. 1997a. Die cymbelloiden Diatomeen: eine monographie der weltweit bekannten taxa. I Allgemeines und Encyonema Part. Bibliotheca Diatomologica 36: 1-382.

Krammer, K. 1997b. Die cymbelloiden Diatomeen: eine monographie der weltweit bekannten taxa. II Encyonema Part., Encyonopsis and Cymbellopsis. Bibliotheca Diatomologica 37: 1-469.

Krammer, K. \& Lange-Bertalot, H. 1986. Bacillariophyceae: Naviculaceae. In: Ettl, H.; Gerloff, J.; Heynig, H. \& Mollenhauer, D. (eds.). Sübwasserflora von Mitteleuropa 2(1): 1-876.

Krammer, K. \& Lange-Bertalot, H. 1988. Bacillariophyceae: Bacillariaceae, Epithemiaceae, Surirellaceae. In: Ettl, H.; Gerloff, J.; Heynig, H. \& Mollenhauer, D. (eds.). Sübwasserflora von Mitteleuropa 2(2): 1-596.

Krammer, K. \& Lange-Bertalot, H. 1991. Bacillariophyceae: Centrales, Fragilariaceae, Eunotiaceae. In: Ettl, H.; Gerloff, J.; Heynig, H. \& Mollenhauer, D. (eds.). Sübwasserflora von Mitteleuropa 2(3): 1-576.

Krammer, K. \& Lange-Bertalot, H. 1991. Bacillariophyceae Achnanthaceae. Kritische Ergänzungen zu Navicula (Lineolatae) und Gomphonema. In: Ettl, H.; Gerloff, J.; Heynig, H. \& Mollenhauer, D. (eds.).Sübwasserflora von Mitteleuropa 2(4):1-437.

Landucci, M.; Ludwig, T.A.V. 2005. Diatomáceas de rios da bacia hidrigráfica Litorânea, PR, Brasil: Coscinodiscophyceae e Feagilariophyceae. Acta Botanica Brasilica 19(2): 345-357.

Lange-Bertalot, H. 1999. Neue Kombinationen von Taxa aus Achnanthes Bory (sensu lato). Iconographia Diatomologica 6: 276-289.

Lange-Bertalot, H. \& Moser, G. 1994. Brachysira. Monographie der Gattung. Bibliotheca Diatomologica 29: 1-212.

Lange-Bertalot, H. \& Metzeltin, D. 1996. Indicators of oligotrophy-800 taxa representative of three ecologically distinct lake types, Carbonate
buffered-Oligodystrophic-Weakly buffered soft water. Iconographia Diatomologica 2: 1-390.

Lange-Bertalot, H.; Metzeltin, D. \& Witkowski, A. 1996. Hippodonta gen. nov.-Umschreibung und begründung einer Gattung der Naviculaceae. Iconographia Diatomologica 4: 247-275.

Leandrini, J.A.; Moreira-Filho, H.; Rodrigues, L. 2002. Espécies perifíticas de Navicula Bory de dois sistemas lóticos do município de Maringá, estado do Paraná, Brasil. Hoehnea 29(1): 49-56.

Lecointe, C.; Coste, M. \& Prygiel, J. 1993. "Omnidia": software for taxonomy, calculation of diatom index and inventories management. Hydrobiologia 260/770: 509-513.

Levkov, Z.; Krstic, S.; Metzeltin, D. \& Nakov, T. 2007. Diatoms of Lakes Prespa and Ohrid. Iconographia Diatomologica 16: 613.

Lobo, E.A.; Leighton, G. 1986. Estructuras comunitarias de las fitocenosis planctonicas de los sistemas de desembocaduras de rios y esteros de la zona central de Chile. Revista de Biologia Marinha, Valparaíso, 22(1): 1-29.

Lobo, E.A.; Callegaro, V.L.M. \& Bender, E.P. 2002. Utilização de algas diatomáceas epilíticas como indicadores da qualidade da água em rios e arroios da região hodrográfica do Guaíba, RS, Brasil. Santa Cruz do Sul, EDUNISC.

Ludwig, T.A.V.; Bigunas, P.I.T.; Neiva, T.F.; Coquemala, V.; Piccinini, C. 2005. Diatomáceas (Ochrophyta) dos lagos do Jardim Botânico. Revista do Museu Nacional do Rio de Janeiro, série livros, 10 301-323.

Ludwig, T.A.V.; Tremarin, P.I.; Becker, V. \& Torgan, L.C. 2008. Thallasiosira rudis sp. nov. (Coscinodiscophyceae): a new freshwater species. Diatom Research 23(2): 389-400.

Mann, D.G.; Macdonald, S.M.; Bayer, M.M.; Droop, S.J.M.; Chepurnov, V.A.; Loke, R.E.; Ciobanu, A. \& Du Buf, J.M.H. 2004. The Sellaphora pupula species complex (Bacillariophyceae): morphometric analysis, ultraestructure and mating data provide evidence for five new species. Phycologia 43(4): 459-482.

Metzeltin, D. \& Lange-Bertalot, H. 1998. Tropical diatoms of South America I. About 700 predominantly rarely known or new taxa representative of the neotropical flora. In: Lange-Bertalot, H. (Ed.). Iconographia Diatomologica 5: 1-695.

Metzeltin, D. \& Lange-Bertalot, H. 2002. Diatoms from the "Island Continent" Madagascar. In: Bertalot, H. (Ed.). Iconographia Diatomologica 11: 1-286.

Metzeltin, D. \& Lange-Bertalot, H. 2005. Diatoms of Uruguay. Compared with other taxa from South América and elsewhere. In: Lange-Bertalot, H. (Ed.). Iconographia Diatomologica 15: 1-736.

Metzeltin, D. \& Lange-Bertalot, H. 2007. Tropical Diatoms of South America II. Special remarks on biogeographic disjunction. In: LangeBertalot, H. (Ed.). Iconographia Diatomologica 18: 1-877.

Moreira-Filho, H. \& Valente Moreira, I.M. 1981. Avaliação taxonômica e ecológica das diatomáceas (Bacillariophyceae) epífitas em algas pluricelulares obtidas nos litorais dos Estados do Paraná, Santa Catarina e São Paulo. Boletim do Museu Botânica Municipal, Curitiba, 47: 1-17.

Moser, G.; Lange-Bertalot, H.; Metzeltin, D. 1998. Insel der Endemiten. Geobotanisches Phänomen Neukaledonien. Bibliotheca Diatomologica 38: $1-464$.

Patrick, R. \& Reimer, C.W. 1966. The diatoms of United States. Philadelphia: Academy of Natural Sciences, 1: 1-688.

Patrick, R. \& Reimer, C.W. 1975. The diatoms of United States: Exclusive of Alaska and Hawaii. Philadelphia, Academy of Natural Sciences. v. 2: 1-213.

Potapova, M. \& Charles, D.F. 2007. Diatom metrics for monitoring eutrophication in rivers of the United States. Ecological Indicators 7: 48-70.

Reichardt, E. 1999. Zur Revision der Gattung Gomphonema. Die Arten um $G$. affine/insigne, $G$. angustatum/micropus, G. acuminatum sowie gomphonemoide Diatomeen aus dem Oberoligozän in Böhmen. In: H. Lange-Bertalot (ed.), Iconographia Diatomologica 8: 1-206.

Reichardt, E. 2004. Eine bemerkenswerte Diatomeenassoziation in einem Queehabitat im Grazer Bergland, Österreich. Ein Beitrag zur Kenntnis seltener und wenig bekannter Diatomeen. In: H. Lange-Bertalot (ed.), Iconographia Diatomologica 13: 419-479. 
Reichardt, E. 1995. Die Diatomeen (Bacillariophyceae) in Ehrenbergs Material von Cayenne, Guyana Gallica. Iconografia Diatomologica 1: $1-49$.

Reichardt, E. 2001. Revision der Arten um Gomphonema truncatum und G. capitatum. Pp. 187-224. In: Jahn, R., Kociolek, J.P., Witkowski, A. \& Compère, P. (eds). Studies on Diatoms. A.R.G. Gantnter Verlag K.G. Ruggell.

Reichardt, E. \& Lange-Bertalot, H. 1991. Taxonomische Revision des Artenkomplexes um Gomphonema angustum, G. dichotomum, G. intricatum, G. vibrio und ahnliche Taxa (Bacillariophyceae). Nova Hedwigia 53(3-4):519-544.

Rodrigues, L. \& Bicudo, D.C. 2001. Similarity among periphyton algal communities in a lentic-lotic gradient of the upper of Paraná river floodplain, Brazil. Revista Brasileira de Botânica 24(3): 235-248.

Rott, E.; Duthie, H.C.; Pipp, E. 1998. Monitoring oganic pollution and eutrophication in the Grand River, Ontario, by means of diatoms. Cannadian Journal of Fisheries and Aquatic Sciences 55: 1443-1453.

Round, F.E.; Crawford, R.M. \& Mann, D.G. 1990. The diatoms: biology and morphology of the genera. New York, Cambridge University Press.

Rumrich, U., Lange-Bertalot, H. \& Rumrich, M. 2000. Diatoms of the Andes. From Venezuela to Patagonia/Tierra del Fuego and two additional contributions. In: Lange-Bertalot, H. (Ed.). Iconographia Diatomologica 9: 1-673.

Schiefele, S. \& Shreiner, C. 1991. Use of diatoms for monitoring nutrients enrichment, acidification and impact of salt in rivers in Germany and Austria. Pp. 103-110. In: Whitton, B.A.; Rott, E. \& Friedrich, G. (Eds.). Use of algae for monitoring rivers. Düsseldorf, Institut für Botanik.

Simonsen, R. 1974. The diatom plankton of the Indian Ocean Expedition of R/V "Meteor", 1964-65 Meteor. Forschungsergebnisse Reihe D-Biologie 19: 1-66.
Simonsen, R. 1987. Atlas and Catalogue of the Diatom types of Friedrich Hustedt 3: 582-583. Berlin, J. Cramer.

Stenger-Kovács, C.; Buczkó, K.; Hajnal, E. \& Padisák, J. 2007. Epiphytic, littoral diatoms as bioindicators of shallow lake trophic status: Trophic diatom index for lakes (TDIL) developed in Hungary. Hydrobiologia 589: 141-154

Stoermer, E.F. \& Smol, J.P. 1999. The diatoms: applications for the environmental and earth sciences. Cambridge, University Press.

Straškraba, M. \& Tundisi, J.G. 2000. Diretrizes para o gerenciamento de lagos: Gerenciamento da qualidade da água de represas. São Carlos, ILEC, IIE.

Toledo JR., A.P.; Talarico, M.; Chinez, S.J. \& Agudo, E.G. 1983. A aplicação de modelos simplificados para a avaliação e processo de eutrofização em lagos e reservatórios tropicais. Pp. 1-34. In: Anais do $1^{\circ}$ Congresso Brasileiro de Engenharia Sanitária.

Torrisi, M; Rimet, F.; Cauchie, H.M.; Hoffmann, L. \& Ector, L. 2006. Bioindication par les diatomées épilithiques et epiphytes dans la rivière Sûre (Luxembourg). Belgian Journal of Botany 139(1): 39-48.

Tremarin, P.I.; Ludwig, T.A.V. \& Moreira-Filho, H. 2008. Eunotia Ehrnberg (Bacillariophyceae) do rio Guaraguaçu, litoral do Paraná. Acta Botanica Brasilica 22(3): 845-862.

Tuji, A. \& Williams, D.M. 2007. Type examination of Japanese diatoms described by Friedrich Meister (1913) from lakes Suwa. Bulletin of the National Science Museum. Tokyo, Ser. B, 33(2): 69-79.

Tuji, A. \& Houki, A. 2004. Taxonomy, ultrastructure, and biogeography of the Aulacoseira subartica species complex. Bulletin of the National Science Museum. Tokyo, Ser. B, 30(2): 35-54.

van Dam, H.; Mertens, A. \& Sinkeldam, J. 1994. A coded checklist and ecological indicator values of freshwater diatoms from the Netherlands. Netherlands Journal of Aquatic Ecology 28(1): 117-133.

Yang, J.R. \& Dickman, M. 1993. Diatoms as indicators of lake trophic status in central Ontario, Canada. Diatom Research 8(1): 179-193.

Versão eletrônica do artigo em www.scielo.br/abb e http://www.botanica.org.br/acta/ojs 